\title{
Simulations of Trade Wind Cumuli under a Strong Inversion
}

\author{
Buorn Stevens,* Andrew S. Ackerman, ${ }^{+}$Bruce A. Albrecht, ${ }^{\#}$ Andrew R. Brown, $@$ \\ Andreas Chlond, \& Joan Cuxart, ** Peter G. Duynkerke, ${ }^{+}+$David C. Lewellen, \#\# \\ Malcolm K. Macvean, $@$ Roel A. J. Neggers, @ Enrique Sánchez,** A. Pier Siebesma, @ \\ AND DAVID E. STEVEnS \&\& \\ * Department of Atmospheric Sciences, University of California, Los Angeles, Los Angeles, California \\ ${ }^{+}$NASA Ames Research Center, Moffett Field, California \\ ${ }^{*}$ Rosenstiel School of Marine and Atmospheric Science, University of Miami, Miami, Florida \\ ${ }^{\circledR}$ Met Office, Bracknell, Berkshire, United Kingdom \\ ${ }^{\star}$ Max-Planck-Institut für Meteorologie, Hamburg, Germany \\ **Instituto Nacional Meteorologia, Madrid, Spain \\ ${ }^{++}$Institute for Marine and Atmospheric Research, Utrecht, Netherlands \\ \#MAE Department, West Virginia University, Morgantown, West Virginia \\ ${ }^{\circledR}$ Royal Netherlands Meteorological Institute, De Bilt, Netherlands \\ ${ }^{\star}$ Lawrence Livermore National Laboratory, Livermore, California
}

(Manuscript received 6 July 2000, in final form 13 November 2000)

\section{ABSTRACT}

The fifth intercomparison of the Global Water and Energy Experiment Cloud System Studies Working Group 1 is used as a vehicle for better understanding the dynamics of trade wind cumuli capped by a strong inversion. The basis of the intercomparison is 10 simulations by 7 groups. These simulations are supplemented by many further sensitivity studies, including some with very refined grid meshes.

The simulations help illustrate the turbulent dynamics of trade cumuli in such a regime. In many respects the dynamics are similar to those found in many previous simulations of trade cumuli capped by weaker inversions. The principal differences are the extent to which the cloud layer is quasi-steady in the current simulations, evidence of weak countergradient momentum transport within the cloud layer, and the development and influence of an incipient stratiform cloud layer at the top of the cloud layer. Although many elements of the turbulent structure (including the wind profiles, the evolution of cloud-base height, the statistics of the subcloud layer, and the nature of mixing in the lower and middle parts of the cloud layer) are robustly predicted, the representation of the stratiform cloud amount by the different simulations is remarkably sensitive to a number of factors. Chief among these are differences between numerical algorithms. These sensitivities persist even among simulations on relatively refined grid meshes. Part of this sensitivity is attributed to a physically realistic positive radiative feedback, whereby a propensity toward higher cloud fractions in any given simulation is amplified by longwave radiative cooling.

The simulations also provide new insight into the dynamics of the transition layer at cloud base. In accord with observations, the simulations predict that this layer is most identifiable in terms of moisture variances and gradients. The simulations help illustrate the highly variable (in both height and thickness) nature of the transition layer, and we speculate that this variability helps regulate convection.

Lastly the simulations are used to help evaluate simple models of trade wind boundary layers. In accord with previous studies, mass-flux models well represent the dynamics of the cloud layer, while mixing-length models well represent the subcloud layer. The development of the stratiform cloud layer is not, however, captured by the mass-flux models. The simulations indicate that future theoretical research needs to focus on interface rules, whereby the cloud layer is coupled to the subcloud layer below and the free atmosphere above. Future observational studies of this regime would be of most benefit if they could provide robust cloud statistics as a function of mean environmental conditions.

\section{Introduction}

The low-level trade wind regime has been recognized as a structural component of the general circulation for

Corresponding author address: Bjorn Stevens, Department of Atmospheric Sciences, University of California, Los Angeles, 405 Hilgard Avenue, Box 951565, Los Angeles, CA 90095-1565. E-mail: bstevens@atmos.ucla.edu at least half a century (von Ficker 1936; Riehl et al. 1951). In recent years the earlier diagnostic studies have been supplemented by theoretical studies and numerical experiments which further show that large-scale circulations are sensitive to the representation of the trade wind regimes and their associated low-level clouds (e.g., Tiedtke et al. 1988). Moist convective processes in the trades are important not only because of their role in 
mediating the transfer of latent heat from the ocean into the atmosphere, but also because of the direct radiative effect of the clouds on both the radiative budget at the top of the atmosphere and the surface energy budget. Because low clouds in trade wind regimes span vast areas of the World Ocean, more recent interest in these regimes has been fueled by a desire to understand how they might respond to the changing composition of the atmosphere (e.g., Albrecht 1989; Ackerman et al. 1993, 2000, hereafter ACK).

Broadly speaking moist convective circulations in the trades can be categorized into three regimes: an upstream regime with large cloud fractions in a shallow planetary boundary layer $(\mathrm{PBL})^{1}$ capped by a strong inversion, a downstream regime with low cloud fractions in a deeper PBL capped by a relatively weak inversion, and an intermediate regime. Most prior studies, including the two previous intercomparison studies of the GEWEX (Global Water and Energy Experiment) Cloud System Studies Working Group One (GCSSWG1) have focused on either the upstream or downstream regime, or on how the transition between regimes takes place. To our knowledge this study is the first to focus on the quasi-steady structure of the intermediate regime.

Apart from the fact that this regime has received relatively little attention, there are other motivations for studying the intermediate regime. For one, changes in the trade wind boundary layer cloud regimes are largely thought to manifest themselves in the intermediate regime. Another motivation for studying this regime is to evaluate whether ideas and concepts developed through a study of the terminal regimes can be extended to (or blended in) the intermediate regimes. In particular there was an interest in knowing to what extent mass-fluxbased scaling relationships, developed on the basis of simulations of shallow cumulus with relatively low cloud fractions (cf. Siebesma and Cuijpers 1995; Siebesma et al. 2000, manuscript submitted to Bull. Amer. Meteor. Sci., hereafter SIE), can be extended to regimes with larger cloud fractions. Also to the extent that this larger cloud fraction manifests itself as stratiform cloudiness at the top of the cloud layer there arises the question as to how best to think about this stratiform component of the cloud system. With these motivations in mind it was decided to base the fifth intercomparison of the GCSS-WG1 on simulations of the trade wind boundary layer as observed during the Atlantic Trade Wind Experiment (ATEX).

This paper reports on the results of this intercomparison. Specifically, we are interested in answering some of the following questions. What is the basic structure and what are the key sensitivities of the (simulated) intermediate regime? What aspects of the simulations

\footnotetext{
${ }^{1}$ For reasons that shall become obvious (cf., Augstein et al. 1974) we define the PBL as that region of the atmosphere extending from the surface to the base of the trade-inversion.
}

are most robust, and where is further refinement required? What sorts of measurements are necessary to better constrain the simulations, and what key parameters appear to control the evolution of the cloud layer? And lastly, how well are simple models able to characterize the simulated dynamics? For the most part these questions are addressed in the body of this paper, subsequent to our presentation of the case and the participant simulations.

\section{The Atlantic Trade Wind Experiment (ATEX)}

ATEX took place in February 1969 and is thoroughly described in a number of papers by the original participants (e.g., Augstein et al. 1973, 1974; Brümmer et al. 1974). It was based on a flotilla of three ships that drifted for nearly 3 weeks in the Atlantic northeast trade wind region (near $12^{\circ} \mathrm{N}, 35^{\circ} \mathrm{W}$ ). The ships could be roughly associated with the vertices of an equilateral triangle with sides of $750 \mathrm{~km}$. At each ship intensive aerological observations were concentrated in the lower $4 \mathrm{~km}$ of the atmosphere with routine deck-level observations every $90 \mathrm{~min}$ as well as radiosonde and radar wind observations at 180-min intervals. Buoy measurements of surface fluxes supplemented the deck measurements. Subsets of ships assembled at three different times "for the purpose of intercomparisons and calibration," which was facilitated by the presence of a fourth ship steaming through the triangle during the first part of the experiment (Augstein et al. 1973). Although the experiment appears to have generated an unprecedented dataset for its time, our study is largely based on secondary data sources (i.e., analyzed datasets that have appeared in the literature).

\section{a. Initial data, boundary conditions, and forcings}

The initial $\left(t_{0}=0\right)$ data for the simulations are drawn from the first part of the experiment, during which time the ship-triangle was embedded in what Augstein et al. (1973) describe as "a nearly classical trade-wind situation." The temperature and humidity profiles are based on sounding data taken over 5 days (between 7 and 12 February 1969) from the northernmost ship of the flotilla, the R/V Planet. The data were first composited using a subjective technique that preserved the transition layer at $z=h$ and the trade inversion at $z=z_{i}$. The initial thermodynamic profiles are plotted in Fig. 1. The main differences between the composite R/V Planet sounding (also plotted) and that actually used are the simplification to the trade-inversion structure and the slight moistening of the free troposphere. The differences between the initial data and the more downstream [i.e., the Meteor and the Barbados Oceanographic and Meteorological Experiment (BOMEX) (SIE)] soundings largely reflects differences in the underlying SSTs. Given the similar free-tropospheric thermal structure and commensurate values of $z_{i}$, the upstream soundings nec- 

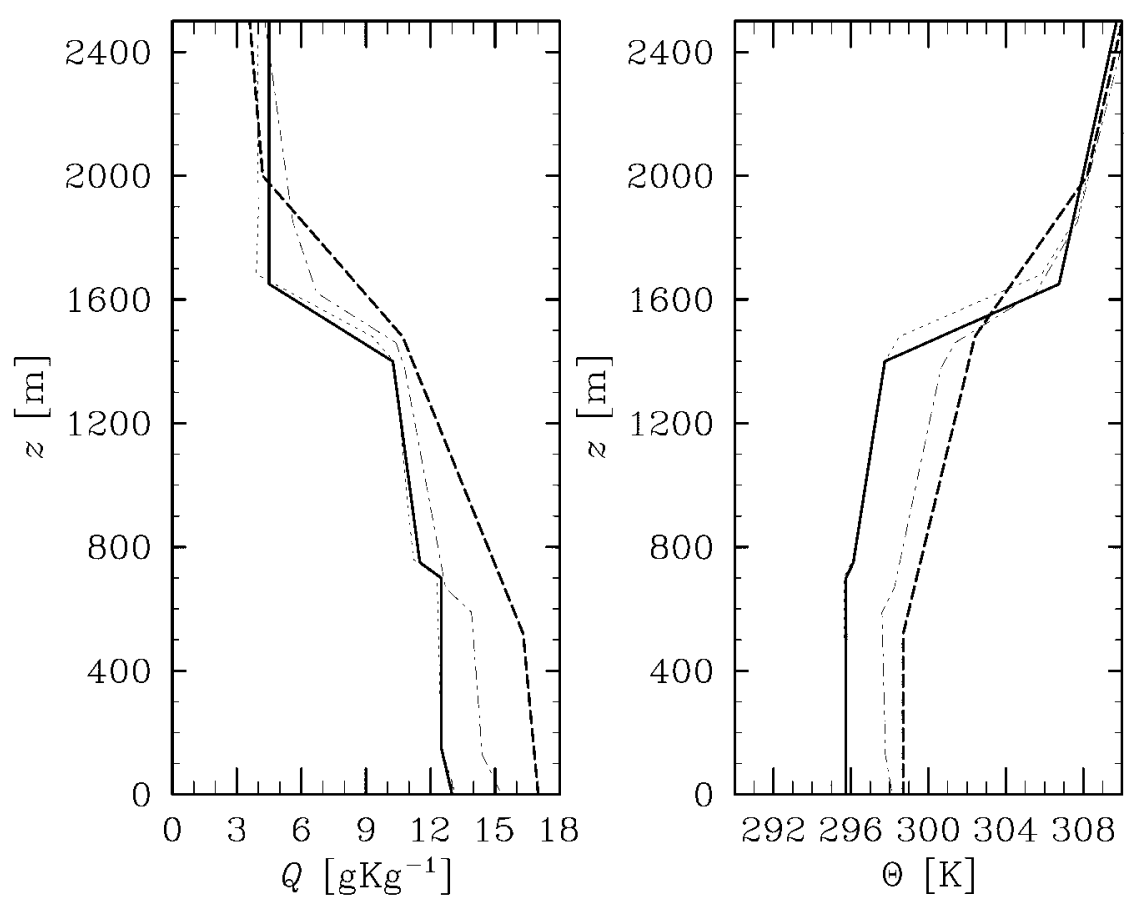

FIG. 1. Profiles of total-water mixing ratio $(Q)$ and potential temperature $(\Theta)$ : initial conditions (solid line), composite R/V Planet sounding (thin dotted), composite R/V Meteor sounding (thin dash-dot), initial conditions for BOMEX simulations (thick dashed).

essarily (insofar as SSTs increase downstream) have a stronger capping inversion. A further difference between the ATEX and the BOMEX soundings is that in the former the compositing procedure preserved the transition layer at the base of the cloud layer. The importance of this modification (and the transition layer in general) is a point we return to later.

The initial winds and their geostrophic values are drawn from published analyses (Brümmer et al. 1974; Augstein et al. 1974). The latter are presented, along with the initial thermodynamic sounding, in Table 1. The geostrophic winds are mostly easterly and modestly baroclinic. In rough accord with the published profiles (Augstein et al. 1974, their Fig. 9; Brümmer et al. 1974, their Fig. 2) the initial winds were prescibed

$$
\begin{aligned}
& u\left(x, y, z, t_{0}\right)=\max \left[U_{g}(z),-8\right], \\
& v\left(x, y, z, t_{0}\right)=V_{g}(z) .
\end{aligned}
$$

TABLE 1. Thermodynamic profiles at $t_{0}$ and geostrophic wind at selected heights. Linear profiles are assumed between these heights.

\begin{tabular}{ccccc}
\hline \hline & & $\begin{array}{c}Q \\
\left(\mathrm{~g} \mathrm{~kg}^{-1}\right)\end{array}$ & $\begin{array}{c}U_{g} \\
\left(\mathrm{~m} \mathrm{~s}^{-1}\right)\end{array}$ & $\begin{array}{c}V_{g} \\
\left(\mathrm{~m} \mathrm{~s}^{-1}\right)\end{array}$ \\
\hline 0 & $\Theta(\mathrm{K})$ & 13.00 & -11.00 & -2.00 \\
150 & 295.750 & 12.50 & -10.55 & -1.90 \\
700 & 295.750 & 12.50 & -8.90 & -1.10 \\
750 & 296.125 & 11.50 & -8.75 & -1.00 \\
1400 & 297.750 & 10.25 & -6.80 & -0.14 \\
1650 & 306.750 & 4.50 & -5.75 & 0.18 \\
4000 & 314.975 & 4.50 & 1.00 & 2.75 \\
\hline
\end{tabular}

The Coriolis parameter was specified to a value corresponding to $15^{\circ} \mathrm{N}$.

In accord with previous intercomparison studies (e.g., Duynkerke et al. 2000; SIE), $u_{*}$ (the surface friction velocity) was prescribed a value of 0.3 . To ensure that the underlying surface fluxes were consistent with an underlying sea surface, SSTs of $298 \mathrm{~K}$ were specified and surface fluxes of heat and moisture were calculated using a simple bulk aerodynamic formula. In retrospect fixing the momentum but not the heat fluxes is somewhat artificial, a point we return to subsequently. To account for the fact that different models might place their first thermodynamic level at varying heights, the bulk exchange coefficient for heat and moisture was corrected assuming neutral stability:

$$
C_{h}=C_{10}\left[\ln \left(\frac{10}{z_{0}}\right) / \ln \left(\frac{z}{z_{0}}\right)\right]^{2},
$$

with the roughness height $z_{0}=0.015 \mathrm{~cm}$ and the exchange coefficient $\mathcal{C}_{10}=0.0013$.

For $t>t_{0}+5400 \mathrm{~s}$ forcings associated with hypothetical large-scale processes are specified relative to $z_{i}$ (before this time no forcings other than surface fluxes were specified). For these purposes $z_{i}$ is defined to be the spatial-mean height of the $6.5 \mathrm{~g} \mathrm{~kg}^{-1}$ total-water mixing ratio contour. This contour was somewhat arbitrarily chosen because it coincides with the region of strong static stability within the trade inversion. Largescale advective tendencies due to subsidence also are imposed based on the value of the local gradients and 

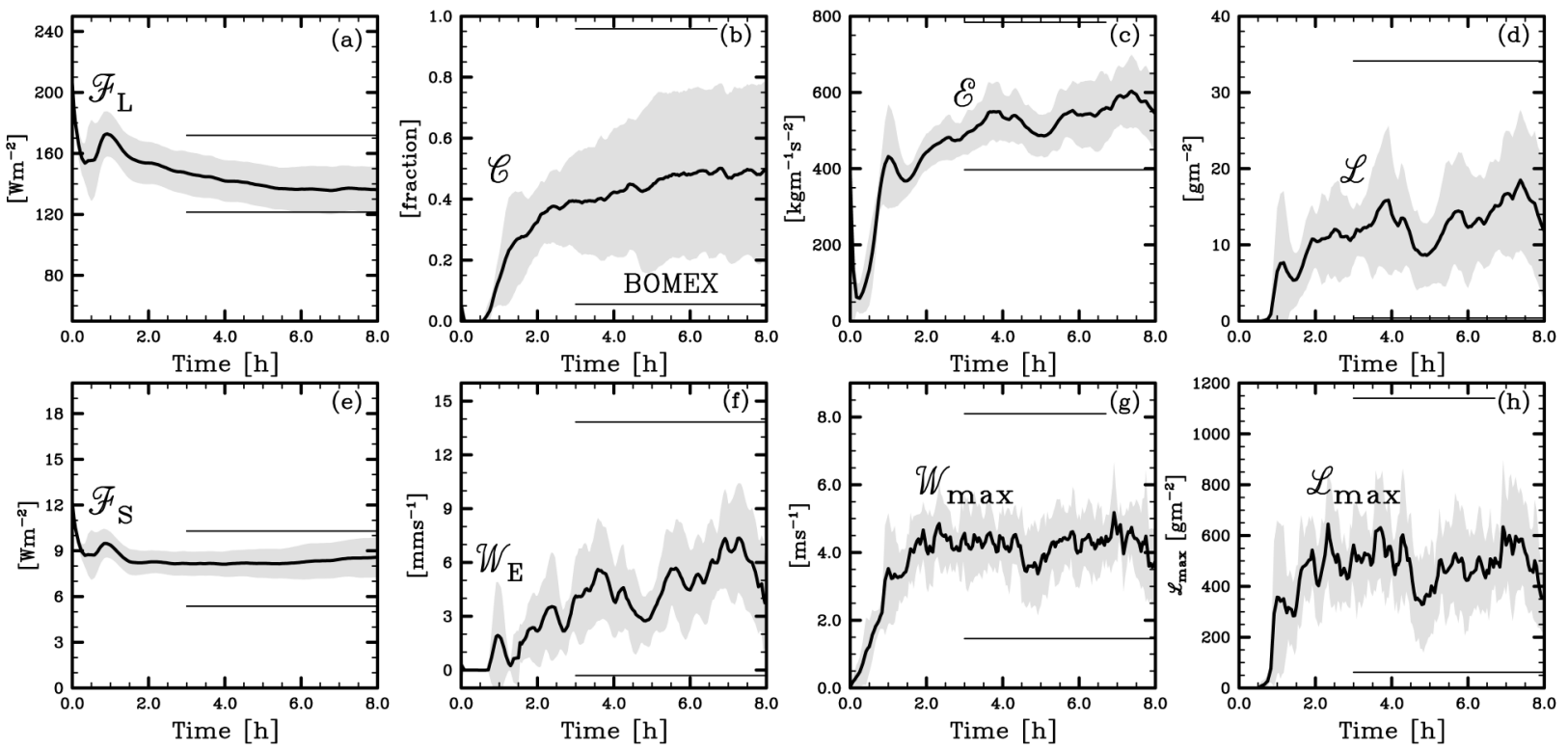

FIG. 2. Time series data. Solid line is average of 10 simulations, band is of width $2 s_{\phi}$ centered at the population mean. Solid horizontal lines delineate extrema during analysis period for all simulations. (a) Surface latent-heat flux $\mathcal{F}_{L}$, (b) fraction of grid columns with liquid water $\mathcal{C}$, (c) Vertically integrated total kinetic energy $\mathcal{E}$, (d) Domain-averaged liquid water path $\mathcal{L}$, (e) surface sensible heat flux $\mathcal{F}_{S}$, (f) mean vertical velocity of $q_{t}=6.5 \mathrm{~g} \mathrm{~kg}^{-1}$ contour $\mathcal{W}_{E},(\mathrm{~g})$ maximum vertical velocity in the domain $\mathcal{W}_{\max }$, (h) maximum liquid water path in a model column $\mathcal{L}_{\max }$. Also indicated by the acronym BOMEX in the cloud-fraction plot is the approximate span of cloud fractions derived from simulations of trade cumulus as observed during BOMEX.

a specified subsidence velocity that varies linearly between 0 at the surface and $6.5 \mathrm{~mm} \mathrm{~s}^{-1}$ at $z_{i}$. Although this value of the large-scale subsidence at $z_{i}$ is arguably too small (cf. Brümmer et al. 1974), it was chosen based on exploratory simulations that suggested that larger values underestimated cloud amount.

The effects of large-scale advective and radiative processes are incorporated by specifying tendencies as follows:

$$
\begin{aligned}
& \left.\frac{d \theta}{d t}\right|_{\mathrm{LS}}=-1.1575 \times 10^{-5}\left(3-\frac{z}{z_{i}}\right) \mathrm{K} \mathrm{s}^{-1}, \\
& \left.\frac{d q_{t}}{d t}\right|_{\mathrm{LS}}=-1.58 \times 10^{-8}\left(1-\frac{z}{z_{i}}\right) \mathrm{s}^{-1},
\end{aligned}
$$

for $z<z_{i}$. These large-scale tendencies were loosely based on observations summarized by Tiedtke (1989). Above the trade inversion the sum of these tendencies and those due to large-scale subsidence was linearly reduced to zero over a depth of $300 \mathrm{~m}$. This tapering above $z_{i}$ was done to enforce an exact balance between radiative cooling and large-scale advection in the free atmosphere.

In a further departure from simulations of the downstream trade wind regime (e.g., BOMEX) up to $74 \mathrm{~W}$ $\mathrm{m}^{-2}$ of radiative cooling are allowed to occur if liquid water is present in sufficient amount. The cooling is calculated by allowing heat to be radiated from the saturated regions of the flow as follows:

$$
\begin{aligned}
& F_{\text {rad }}\left(x, y, z, t ; F_{0}, \kappa\right) \\
& \quad=F_{0} \exp \left[-\kappa \int_{z}^{\infty} \rho\left(z^{\prime}\right) q_{l}\left(z^{\prime}, x, y, t\right) d z^{\prime}\right],
\end{aligned}
$$

where $\rho$ is the basic-state density and $q_{l}$ is the liquid water mixing ratio. The parameters, $F_{0}=74 \mathrm{~W} \mathrm{~m}^{-2}$ and $\kappa=130 \mathrm{~m} \mathrm{~kg}^{-1}$, are in accord with previous use of the above formula (e.g., Duynkerke et al. 2000). Although such a simple model is clearly unrealistic in many respects, it is efficient and well represents the firstorder effect wherein clouds efficiently concentrate the radiative cooling of the $\mathrm{PBL}$ in a thin layer near cloud top. Most importantly, it ensures consistency in the governing equation set used by the different intercomparison participants.

Although we have made an effort to incorporate a number of physical forcings, we neglect the role of precipitation. One could argue that precipitation plays a decisive role in circulations such as those discussed here. Thus its neglect is justified not by its lack of potential importance, but rather because an attractive first step in studying this cloud regime is to first understand the behavior and sensitives of the nonprecipitating idealization.

Although the initial data and the forcings are based on an observed case, our intent is not to evaluate the large eddy simulation (LES) based on the data. The observed case was used for the initialization primarily to place the simulations in a plausible regime. While it 
TABLE 2. Participating simulations. Except for DHARMA the simulation names are based on acronyms that reflect the name of the participating scientists' institution. In the cloud-fraction field both the average over the analysis period and the average over the last $2 \mathrm{~h}$ are given. For the subgrid-scale (SGS) models T refers to TKE schemes, while S refers to Smagorinsky schemes. In both types of schemes details of implementation and the model of the length scale varies considerably among groups. Advection schemes are classified as to whether they are centered (C) or monotone (M). Both the UKMO and DHARMA models are distinct in that their monotone schemes are formally multidimensional.

\begin{tabular}{|c|c|c|c|c|c|c|}
\hline $\begin{array}{l}\text { Simulation } \\
\text { name }\end{array}$ & Participating scientists & $\begin{array}{l}\text { Cloud } \\
\text { fraction }\end{array}$ & $\begin{array}{l}\text { SGS } \\
\text { model }\end{array}$ & $\begin{array}{l}\text { Velocity } \\
\text { adv. }\end{array}$ & $\begin{array}{l}\text { Scalar } \\
\text { adv. }\end{array}$ & $\Delta x$ \\
\hline WVU-A & Lewell & $0.17 / 0.14$ & $\mathrm{~T}$ & $\mathrm{C}_{\prime \prime}$ & $\mathrm{M}$ & $100 \mathrm{~m}$ \\
\hline WVU-B & " & $0.39 / 0.39$ & $\mathrm{~T}^{*}$ & $"$ & " & " \\
\hline INM & Sanchez & $0.37 / 0.38$ & $\mathrm{~T}$ & $\mathrm{C}$ & $\mathrm{C}$ & $"$ \\
\hline MPI & Chlond & $0.32 / 0.35$ & $\mathrm{~T}$ & $\mathrm{C}$ & M & $"$ \\
\hline UCLA-A & B. Stevens & $0.22 / 0.23$ & $\mathrm{~S}$ & $\mathrm{C}$ & M & $"$ \\
\hline UCLA-B & $"$ & $0.72 / 0.90$ & $"$ & $"$ & $\mathrm{C}$ & $"$ \\
\hline UKMO-A & Brown & $0.50 / 0.56$ & $\mathrm{~S}$ & $\mathrm{C}$ & M & $"$ \\
\hline UKMO-B & $"$ & $0.75 / 0.75$ & $"$ & M & $"$ & $"$ \\
\hline KNMI & Siebesma and Neggers & $0.26 / 0.27$ & $\mathrm{~T}$ & $\mathrm{C}$ & $\mathrm{C}$ & $"$ \\
\hline DHARMA & Ackerman and D. Stevens & $0.86 / 0.91$ & $\mathrm{~S}$ & M & M & $"$ \\
\hline UCLA-C & B. Stevens & $0.24 / 0.27$ & $\mathrm{~S}$ & $\mathrm{C}$ & M & $50 \mathrm{~m}$ \\
\hline UCLA-D & " & $0.86 / 0.97$ & $\mathrm{~S}$ & $\mathrm{C}$ & $\mathrm{C}$ & " \\
\hline UCLA-E** & $"$ & $0.28 / 0.26$ & $\mathrm{~S}$ & $\mathrm{C}$ & M & $"$ \\
\hline UKMO-C & MacVean & $0.82 / 0.86$ & S & $\mathrm{C}$ & M & $20 \mathrm{~m}$ \\
\hline UKMO-D & $"$ & $0.98 / 0.98$ & $\mathrm{~S}$ & M & M & " \\
\hline
\end{tabular}

* The SGS model used by WVU-B involves the prediction of SGS cloudiness.

** The UCLA-E calculations uses full surface-layer similarity theory to compute the surface fluxes, i.e., $u *$ is not fixed and stability functions are calculated iteratively.

is clearly desirable to test LES using observations, the ATEX data (as are all existing datasets of trade wind boundary layers) are ill-suited to this purpose. The simulations do, however, suggest critical measurements that could be made, and these are outlined in due course.

\section{b. Procedures and participants}

Our evaluation is centered around 10 simulations by seven participating groups. ${ }^{2}$ The simulations are tabulated in Table 2. All simulations from this standard suite were performed with grid spacings of $\Delta x=\Delta y=5 \Delta z$ $=100 \mathrm{~m}$, although some calculations were based on a stretched vertical coordinate above $1750 \mathrm{~m}$. The domain had a vertical extent of about $3000 \mathrm{~m}$ and a horizontal extent of $6400 \mathrm{~m}$. Boundary conditions were periodic in lateral directions, and rigid lids capped the flow above and below. To prevent gravity wave energy from accumulating at, or being reflected from, the upper lid, most simulations included a damping or "sponge" layer near the upper boundary. The symmetry of the initial conditions was broken by introducing zero-mean, pseudorandom, fluctuations in the initialization of $\theta$ and $q_{t}$ below $z=810 \mathrm{~m}$. The amplitudes of these fluctuations were $0.1 \mathrm{~K}$ and $0.025 \mathrm{~g} \mathrm{~kg}^{-1}$, respectively. The integrations were carried out for $8 \mathrm{~h}$ of simulated time and the analysis was over the last five simulated hours. The

\footnotetext{
${ }^{2}$ Although in three instances multiple simulations by a single group are included these represent substantial modification to some element of the flow solver on which the simulation was based, and there is no evidence that including these three additional simulations artificially reduces the spread among the simulations.
}

eddy-turnover time $\tau$ (taken as the ratio of the depth of a layer to the maximum value of $\left(\overline{w^{\prime} w^{\prime}}\right)^{1 / 2}$ within a layer) was approximately 30 and $45 \mathrm{~min}$ for the subcloud and cloud layer, respectively, both of which are consistent with the temporal evolution of the simulations (i.e., anomolies in the turbulent statistics are correlated on these timescales).

All the models used an identical formulation for calculating surface and radiative fluxes. All groups excepting West Virginia University (WVU) used the same saturation vapor pressure formula; fortunately differences between this formula and the one used by the WVU group appear to be negligible. All simulations were based on a staggered (Arakawa C) grid. Excepting the Distributed Hydrodynamic-Aerosol-Radiation-Microphysics Application (DHARMA) [and some implementations of the Met Office (UKMO) model], which used forward-in-time differencing throughout and upwinded momentum (Stevens and Bretherton 1997), all models used centered-in-space (and time) differencing for momentum, although some used upwind methods for scalars. The main differences among the simulations were in terms of how they formed their basic state (anelastic vs Boussinesq), the numerical methods they used to represent spatial differences in advective operators (particularly for scalars), and the manner in which they represented unresolved covariances, that is, their subgrid-scale (SGS) parameterization.

In addition to the standard calculations a number of further simulations were performed. For instance the UKMO group explored the sensitivity of the simulations to a fivefold reduction in horizontal grid spacing as well as to the representation of momentum advection; the 
WVU simulations explored the sensitivity of the simulations to fractional cloudiness parameterizations; and the University of California Los Angeles (UCLA) model was also used to explore resolution sensitivities, scalar advection sensitivities, ensemble statistics, and initial sounding sensitivities, as well as the sensitivity to a wide variety of changes in the SGS model. The ensemble simulations (which are most frequently referred to) consisted of repetitive simulations using the UCLA-A configuration; these simulations differed from one another only in terms of the initial random tickling used to break the symmetry in the initial data. The results from all of the auxiliary simulations will not be discussed systematically, instead they will be used to flavor our analysis of the standard case as appropriate.

Although this study is based on a model intercomparison, preliminary analyses and past experience suggest that there is not a lot to be learned by focusing on the differing performance of individual models. For this reason we concentrate our analysis on the big picture painted by the family of simulations. Nonetheless, by digging a little deeper, and looking at similarities and differences across several models, or the sensitivity of a control calculation to a single change, we are able to arrive at measures of the robustness of LES of the intermediate trade cumulus regime. Such a procedure helps us understand what aspects of LES of this regime might be expected to be reliable, and what aspects clearly are not. The most reliable statistics are then those that we can identify as touchstones for a general theoretical development, as well as a target for both measurements and reproduction by simpler models.

\section{c. Analysis methodology}

The analysis is largely based on two types of fields, scalar time series and profiles. Time series variables may involve point measures, or integrals from a sequence of snapshots, while profiles are averaged in horizontal dimensions and in time. To distinguish spatial (and/or temporal) averaging of a single simulation from averaging across different simulations we use the overbar and angle brackets, respectively. So, for instance, given a set of fields $\Phi_{j}\left(\right.$ or $\left.\bar{\Phi}_{j}\right)$, where $j$ indexes the $N$ simulations that the sample comprises, and $\Phi$ is either a function of the vertical coordinate $z$ or time $t$, we define

$$
\langle\Phi\rangle=\frac{1}{N} \sum_{j=1}^{N} \Phi_{j}
$$

To simplify notation (particularly in regard to the figures) upper case is sometimes used in lieu of an overbar, and script letters may serve as a proxy for angle brackets (i.e., $\langle\bar{a}\rangle=\langle A\rangle=\mathcal{A}$ ).

To best emphasize the collective behavior of the models we generally display the results in terms of a band of width $2 s_{\Phi}$ centered about the mean $\langle\Phi\rangle$. Here $s_{\Phi}$ is the standard deviation of the sample population, that is,

$$
s_{\Phi}^{2}=\frac{1}{N-1} \sum_{j}\left(\Phi_{j}-\langle\Phi\rangle\right)^{2} .
$$

In some cases, good sense dictates the omission of outlying data from the population sample. In such instances the omitted values are either plotted separately or their behavior is noted in the text. Because we understand that the results of individual calculations might be of broader interest, we will be happy to make the statistics from all the simulations available upon request.

\section{Results}

\section{a. Time evolution}

Different measures of the evolution of the simulations are plotted in Fig. 2. By the third hour the flow is relatively stationary. Surface Bowen ratios are about 0.065 , so latent and sensible heat fluxes contribute approximately equally to surface buoyancy fluxes. Time-averaged cloud fractions vary widely among the simulations as do temporal trends. In some cloudiness is increasing, in others it is essentially constant, and in one it even decreases through the analysis period. Nonetheless, all simulations tend to be in the intermediate regime; that is, cloud fractions are between what is commonly observed in the two extreme regimes (i.e., 0.1 and 0.9). Updrafts reach velocities between 4 and $5 \mathrm{~m}$ $\mathrm{s}^{-1}$ and liquid water paths through the cumulus turrets approach $500 \mathrm{~g} \mathrm{~m}^{-2}$, which is about half of the adiabatic value.

Over the analysis period least squares linear fits to the ensemble-average surface fluxes change by less than $10 \%$, and the integrated total kinetic energy $\mathcal{E}$ increases by less than $15 \%$. Cloud measures show more distinct trends: cloud fraction $(C)$, averaged liquid water path $(\mathcal{L})$ and the proxy entrainment rate $\left(\mathcal{W}_{e}\right)$ all increase by $25 \%-30 \%$. Although these trends are significant, we have found no evidence that our findings are impacted by these temporal features.

The coefficient of variation $\mathrm{cv}_{\Phi}=\mathrm{s}_{\Phi} / \bar{\Phi}$ for some field $\Phi$, is essentially a normalized variability, which makes it easier to compare the variability among fields. Values of $\mathrm{cv}_{\Phi}$ range from about 0.1 for the fluxes and integrated turbulent kinetic energy (TKE) to values greater than 0.5 for both $\mathcal{C}$ and $\mathcal{W}_{e}$. For all except the point measures (e.g., $w_{\max }$ and $\mathcal{L}_{\max }$ ) this degree of variability among the simulations is real; that is, it is not simply an artifact due to the aliasing of different phasings in the time variability of different simulations. This degree of variability also turns out to be a factor of 10 larger than the value obtained by comparing an ensemble of simulations from a single code. For instance, while $\mathrm{cv}_{C} \approx 0.5$ for the intercomparison ensemble, for the five-member UCLA-A ensemble $C$ ranged from 0.19 to 0.22 with $\mathrm{cv}_{C}=0.06$. Thus the variations among the simulations truly reflect the sensitivity of the calculations to algorithmic details.

As pointed out above, the simulations were largely 

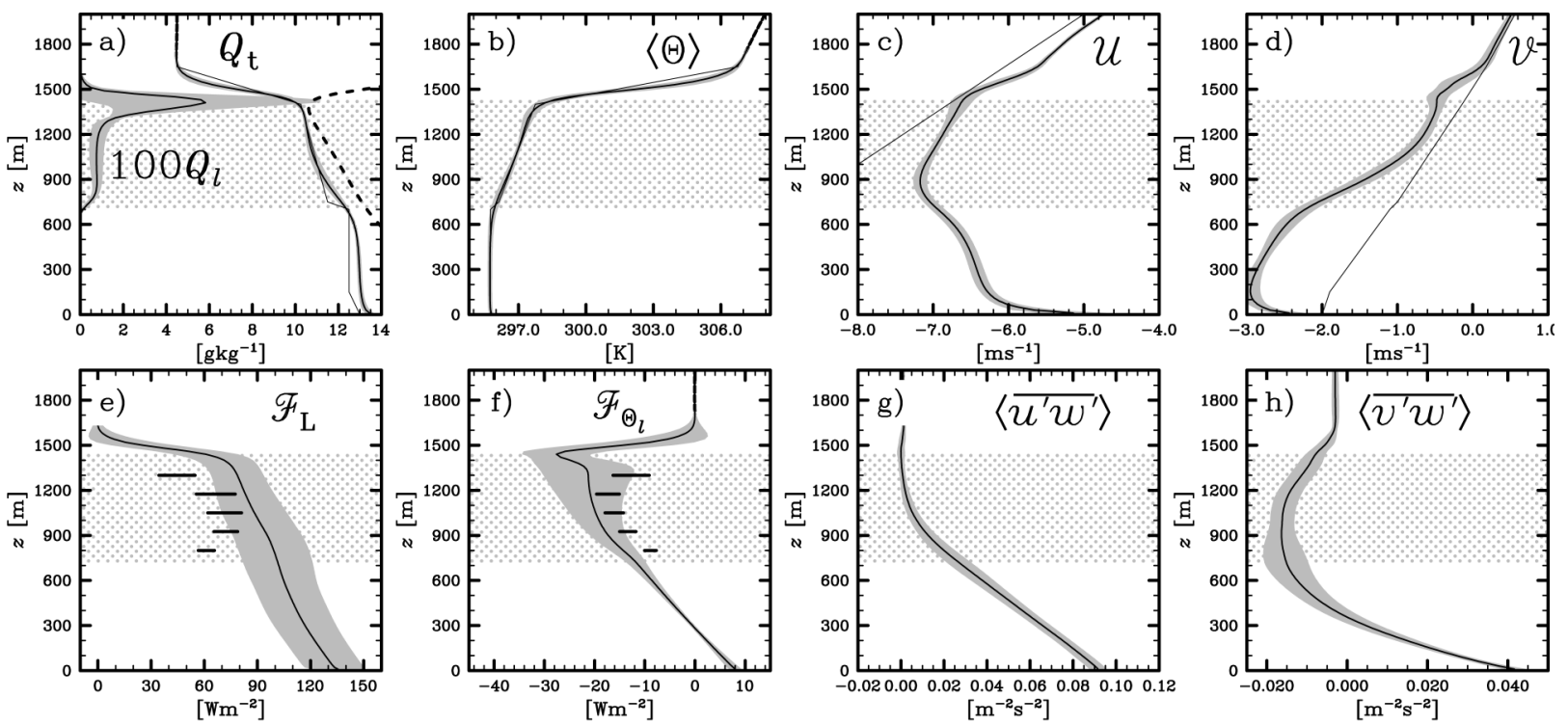

FIG. 3. Mean profiles averaged over analysis period and displayed following format of previous figure. Thin solid lines delineate initial state. Plotted, clockwise from top left are (a) total-water $Q_{t}$ and liquid water $Q_{\downarrow}$ mixing ratios, (b) potential temperature $\Theta$, (c) zonal wind $\mathcal{U}$, (d) meridional wind $\mathcal{V}$, (e) total-water mixing ratio flux $\mathcal{F}_{f}$, (f) liquid water potential temperature flux $\mathcal{F}_{\theta},(\mathrm{g})$ zonal momentum flux, and (h) meridional momentum flux. All the fluxes are the sum of the resolved and SGS fluxes. (e) and (f) The mass-flux estimate of the flux is also shown by the short horizontal lines at five heights [see section $4 \mathrm{~b}(1)$ for details]. (a) The thin dashed line denotes $Q_{s}$ in the cloud layer.

successful in representing a regime with intermediate cloud fractions, although actual values of cloud fraction (and the ensuing domain-averaged liquid water path) varied sharply across the simulations. Albrecht (1991) shows time series of cloud fraction from both the R/V Planet and the R/V Meteor. Over the R/V Planet, whose thermodynamic environment was most commensurate with the specified initial data, cloud fractions varied between 0.1 and 0.9 , with a distinct diurnal cycle and a trend toward lower cloud fractions as the ship drifted over warmer water. Thus while the simulations are broadly consistent with the data, the intercomparison clearly illustrates the difficulty of using LES to quantify relationships between cloud fraction and the large-scale environment - at least in this regime.

\section{b. Mean profiles and fluxes}

With the exception of the velocity deficits (i.e., $u-$ $u_{g}, v-v_{g}$ ) in the subcloud (and to a lesser extent) cloud layer, the evolution of the simulations over $8 \mathrm{~h}$ results in remarkably small changes in the mean state (Fig. 3). This is largely a result of the forcings balancing within the Eulerian domain. The thermodynamic fluxes in Fig. 3 illustrate the tight coupling of the cloud and subcloud layers. To the extent that the boundary layer is considered as a single layer, energetically coupled to the surface on short timescales, the PBL in this regime extends from the surface to the base of the trade inversion (which we denote by $z_{i}$ ) at $1500 \mathrm{~m}$.

On the other hand, both the momentum profiles and fluxes behave distinctly in the cloud versus the subcloud layers. The largest velocity deficits (i.e., departures of the velocities from their geostrophic values) occur in the subcloud layer (i.e., for $z<h$, where $h$ denotes the height of the subcloud layer) and a weak zonal jet develops just above $z=h$. Above this jet (Fig. 3c), zonal velocity gradients reverse, taking on the sign of the gradients of the geostrophic wind. However, the zonal momentum flux (Fig. 3g) does not change sign, which implies a weak countergradient transport of zonal momentum. This locally countergradient flux is consistent with momentum being mixed out of the subcloud layer, as opposed to down the local gradient. The meridional wind (Fig. 3d) has a markedly different structure, tending to peak near the surface with relatively more active momentum transport (Fig. 3h) in the cloud. The greater transport of meridional momentum in the cloud layer is consistent with the somewhat larger differences between the meridional wind (as compared to the zonal wind) in the subcloud versus the cloud layer.

The aforementioned variability in cloud statistics is most evident at cloud top, where both cloud water and cloud fraction (shown later) have global maxima that vary widely. Flow visualization and conditional sampling (also discussed below) indicate that the variability in cloud water largely reflects different predictions of the lifetime (and hence extent) of stratiform detrainment regions associated with cumulus clouds impinging upon the trade inversion. The mean state saturation deficit (i.e., $\bar{q}_{s}-\bar{q}_{t}$ ) is a minimum at about $1400 \mathrm{~m}$, just below the region of maximum liquid water. The tendency for 

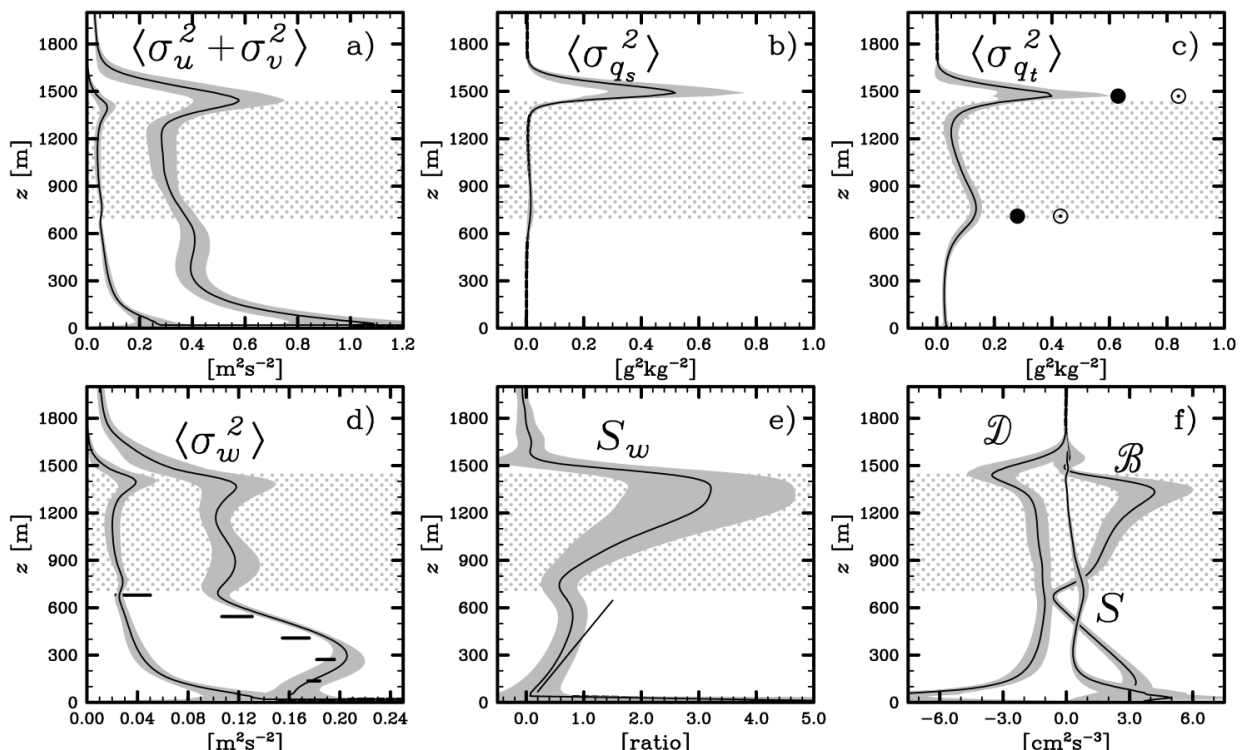

FIG. 4. Higher-order statistics: (a) total horizontal velocity variances (and estimated SGS contribution); (b) saturation mixing-ratio variances; (c) total-water mixing-ratio variances; (d) vertical velocity variances (and estimated SGS contribution), also shown with short horizontal lines are the mixed layer scaling estimates; (e) skewness of the vertical velocity (thin line in subcloud layer denotes expected value for dry convective PBL); and (f) terms in the TKE budget, where $\mathcal{D}, \mathcal{B}$, and $\mathcal{S}$ refer to dissipation, buoyancy, and shear production of TKE, respectively. (b) The WVU computations were not included due to a diagnostic problem in calculating $\sigma_{q}$ in the lowest $500 \mathrm{~m}$. (c) The two calculations (UKMO-B and DHARMA) with forward-in-time (and upwinded) representations of momentum advection are not included in the ensemble statistics, but the local maxima produced by each are indicated separately by the filled circle and circledot, respectively. (d) In the plot of $\sigma_{w}^{2}$ the integrations from the KNMI and INM groups are not included for reasons discussed in the text.

cloud amount to peak slightly above the region of the minimum saturation deficit is probably related to the fact that both $\sigma_{\bar{q}_{t}}$ (the standard deviation among mean values from different simulations) and $\left\langle\sigma_{q_{t}}\right\rangle$ (the mean of the standard deviations of the simulations) have their maxima closer to $1500 \mathrm{~m}$. This suggests that the production of scalar variance at the top of the cumulus layer influences the local cloud amount, a suggestion that receives support from the sensitivity of cloud amount to resolution and SGS parameterizations. For instance, inclusion of an SGS cloud parameterization in the WVU calculations effectively doubles the predicted cloud fraction. Unfortunately, efforts to extract simple relations between cloud amount and second-order statistics (or even layer mean values) are greatly frustrated by the fact that the development of cloud significantly affects the local circulations.

An effort was made to preserve the transition layer in the initial data, as there has been some suggestion (e.g., Garstang and Betts 1974) that it could be dynamically important to the simulations. In Fig. 3 very little evidence of this initially sharp layer remains. To get a better understanding of the statistics of the cloud-base layer we have further examined 10 snapshots. The snapshots were taken from two different times (one at $t=$ $4 \mathrm{~h}$, the other at $t=8 \mathrm{~h}$ ) from each of the five UCLA-
A ensemble members. This analysis indicated that the transition layer is evident if it is based on local absolute humidity gradients, but fluctuations of the transition layer itself make it difficult to identify in layer-averaged quantities. Attempts to identify the transition layer based on local temperature gradients were largely unsuccessful. These findings are consistent with analyses of observational data [which also show that transition layers are most readily identifiable with moisture gradients (Garstang and Betts 1974; Yin and Albrecht 2000)] and are perhaps expected given the respective geometries of the moisture and heat fluxes. By the flux geometry we mean the shape of the flux profile, which is single signed for moisture, but changes sign for heat.

\section{c. Higher-order statistics}

The basic turbulent structure of the PBL is illustrated in Fig. 4. With regard to the structure of the turbulent velocity field we note a clear distinction between the cloud and subcloud layer. As has been noted many times previously (e.g., Garstang and Betts 1974; Sommeria 1976), the subcloud layer follows mixed layer similarity in that it is indistinguishable from simulations of a convective boundary layer.

Ignoring for now the cloud layer, the simulations yield 
$z_{*}=h=680 \mathrm{~m}$ and $Q_{*}=4.71 \times 10^{-4} \mathrm{~m}^{2} \mathrm{~s}^{-3}$ for height and flux scales, respectively. The corresponding convective velocity scale is then $w_{*}=0.68 \mathrm{~m} \mathrm{~s}^{-1}$. Convective scaling based on these parameters predicts that $\sigma_{w}^{2} / w_{*}^{2}$ obtains a maximum value of approximately 0.4 at around $0.4 z_{*}$. Figure 4 also shows the convective scaling predictions for $\sigma_{w}$ and $\mathcal{S}_{w}$ as derived from previous simulations, tank experiments, and field measurements (Schmidt and Schumann 1989). Overall the agreement is rather good, with the greatest disagreement at the top of the subcloud layer, where the interaction with the convective layer prevents $\sigma_{w}^{2}$ from vanishing, which in turn implies smaller values of $\mathcal{S}_{w}$. There is, however, some indication that these departures should be even larger. Analysis of subcloud-layer statistics from the GARP (Global Atmospheric Research Program) Atlantic Tropical Experiment (P. Lemone 2000, personal communication) suggests that above $z / h=0.7$ real subcloud layers have even larger departures from mixed layer scaling.

The cloud layer, on the other hand, is distinctly less energetic than the subcloud layer, although dissipation is marginally enhanced, and is characterized by much more sharply skewed vertical velocity fields. Because $\sigma_{w}$ is relatively flat in the cloud layer, large $\mathcal{S}_{w}$ implies increasing third moments through this layer. In other words, updrafts tend to become increasingly energetic and compact as one moves up through the cloud layer. This behavior is consistent with the decreasing mass flux and increasing cloud-averaged vertical velocities (below $1200 \mathrm{~m}$ ).

Overall, the simulations tend to diverge more in their predictions of the turbulent structure as one moves upward through the cloud layer. Models disagree most just at the base of the trade inversion. At this level there tends to be significant scatter among simulated values of scalar and velocity variances, as well as higher moments. But there is also significant variation within the cloud layer. Comparing these fields with those predicted by the five-member UCLA ensemble indicates that the variability among simulations reflects real differences. The most striking disagreement between simulations tends to be between those with forward-in-time (and spatially upwinded) representations of momentum advection (DHARMA and UKMO-B) and the rest. But these differences may in part result because both the DHARMA and UKMO-B calculations produce substantially more stratiform cloud and thus develop a substantially more stratocumulus-like (larger $\sigma_{w}$ and smaller $\mathcal{S}_{w}$ ) circulation at cloud top.

Some differences were so striking that results from different groups were not included in the ensemble averages. The DHARMA simulation produced estimates of SGS TKE that were an order of magnitude larger at the top of the cloud layer (i.e., $e \approx 3 \mathrm{~m}^{2} \mathrm{~s}^{-2}$ ) than those predicted by other simulations, and an order of magnitude smaller through the body of the boundary layer. Because the momentum advection in this model results in some implicit dissipation, diagnostic estimates of quantities related to the explicit representation of dissipation may be less meaningful. In any case, including results from this model in estimates involving SGS TKE would have grossly overrepresented the spread among the simulations. Both the Royal Netherlands Meteorological Institute (KNMI) and Instituto Nacional Meteorologia (INM) simulations were excluded from the plots of $\sigma_{w}^{2}$. These simulations predicted subcloud maxima of 0.37 and 0.51 , respectively. Although we have ruled out the possibility that these differences are due to a diagnostic error, we have made little progress in further isolating their cause. Whatever the cause of the disagreement, it is startling how little evidence there is of these differences projecting onto other fields.

The tendency of all the simulations to predict a pronounced local maxima in $\sigma_{q_{t}}$ at $z \approx 700 \mathrm{~m}$ (i.e., in Fig. $4 c$ ) is consistent with the sense of the mean profiles and with the transition layer being more identifiable in the humidity field. Consistent with the forcings and the local gradients we find that temperature variances, as represented by $\sigma_{q_{s}}$, tend to be smaller, except at the top of the cloud layer where they are commensurate with $\sigma_{q_{t}}$. Figure $4 \mathrm{c}$ also indicates that those simulations that represent momentum advection using forward-in-time (and upwinded) schemes produce starkly different tracer variance fields. While such differences at the top of the cloud layer are consistent with the predictions of greater cloud fractions, the differences at cloud base are also substantial. Possible causes for these differences are explored in further detail in section $4 \mathrm{a}(1)$.

\section{d. Snapshots and structures}

Our discussion would not be complete without at least some snapshots of the flow field. Figure 5 is constructed from the final time of the UCLA-D simulation. The grid spacing in this calculation has been refined by a factor of 2 in the horizontal. The resulting statistics are similar to those from the UCLA-B calculation, but the snapshots are more pleasing to the eye. The flow from this calculation develops high cloud fractions $(C=0.97$ at the time of the snapshot). While this is uncharacteristically large compared to the lower-resolution simulations, it was chosen because it nicely illustrates the relationships between the cumulus layer and capping stratiform layer. Snapshots from similar simulations but with lower cloud fractions have also been analyzed, and the extent to which they differ from the results presented here is noted in our discussion below.

Figures $5 \mathrm{a}-\mathrm{c}$ illustrate that the flow at $300 \mathrm{~m}$ is organized in streets roughly aligned with the wind (which is oriented at approximately $210^{\circ}$ at this time). As one moves closer to the surface, the spacing between streets becomes progressively smaller. While there is still evidence of the streets at the top of the subcloud layer, they seem to break down into more plumelike structures associated with individual cumulus. The streets become 

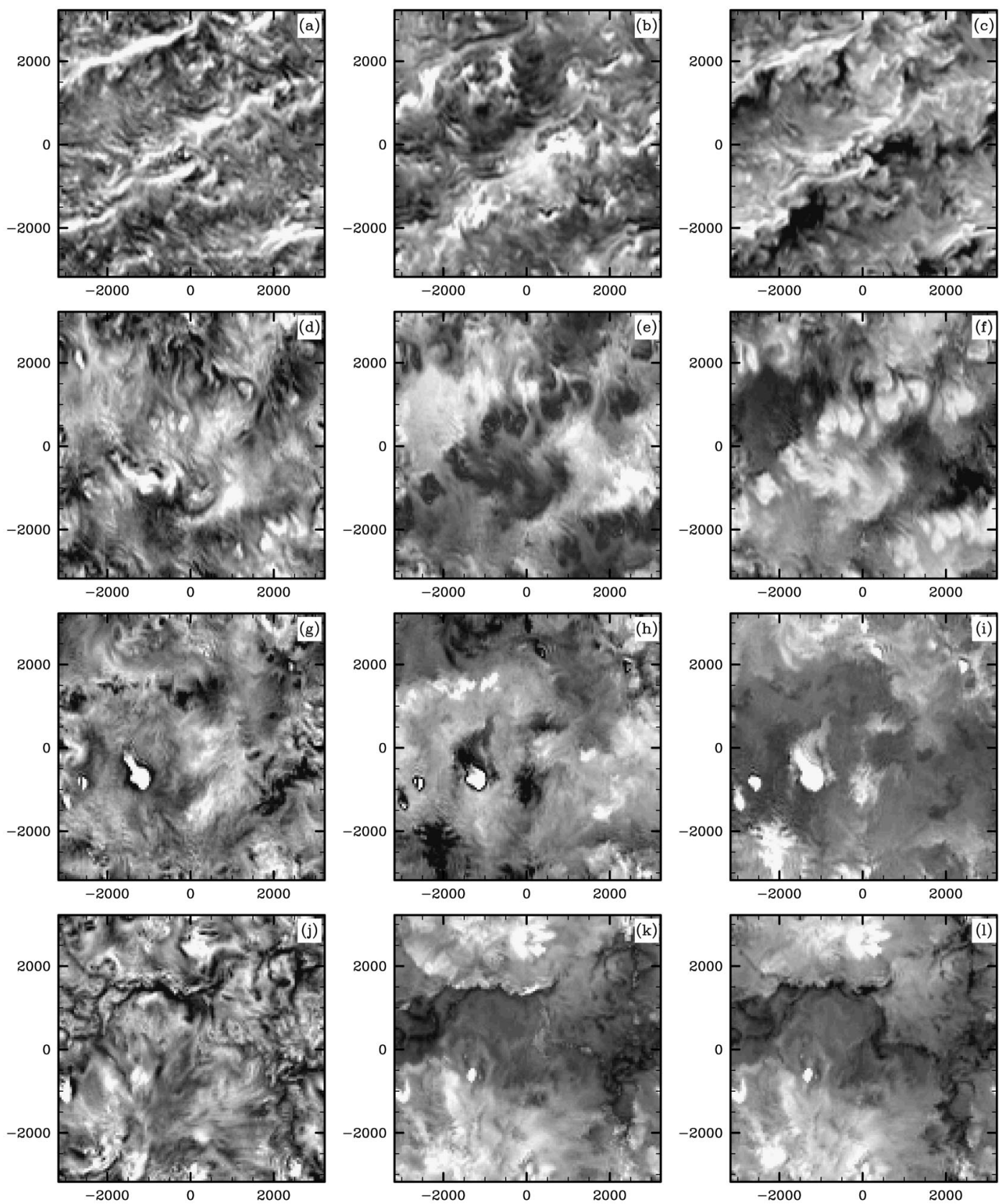

FIG. 5. Plan-view, raster-shaded snapshots of the UCLA-D calculation at $t=8 \mathrm{~h}$. (a)-(c) At $300 \mathrm{~m}$, (d)-(f) at 720 $\mathrm{m},(\mathrm{g})-(\mathrm{i})$ at $1100 \mathrm{~m},(\mathrm{j})-(1)$ at $1400 \mathrm{~m}$. Left column is $w$, center is $\theta$, and right is $q_{\text {. }}$. For some field $\Phi$ the shading uniformly spans $4 \sigma_{\Phi}$, with whiter shades indicating larger values. For $|\Phi|>2 \sigma$, the points are left unshaded, thus whitened areas ringed by black indicate $\Phi<-2 \sigma$ [such as in (h)].

increasingly less evident as one moves up through the cloud layer. At $1400 \mathrm{~m}$, which is at the base of the stratiform cloud layer, there is arguably a single largescale circulation being fed by several convective cells, which covers the entire domain. This is most evident in Figs. 5k-51, which illustrate the $\theta$ and $q_{t}$ fields, respectively. The tendency to produce large-scale circulations is absent, or at least much less evident, in sim- ulations that produce much more modest cloud fractions at the top of the cloud layer.

Flow visualization in the cloud layer (Figs. 5g-i) illustrates the degree to which cumulus turrets depart from the ambient flow. The stark presence of these turrets in both the scalar fields and in $w$ reflects their efficiency in transporting heat and moisture. There is a strong correlation between the circulations in the cloud and sub- 

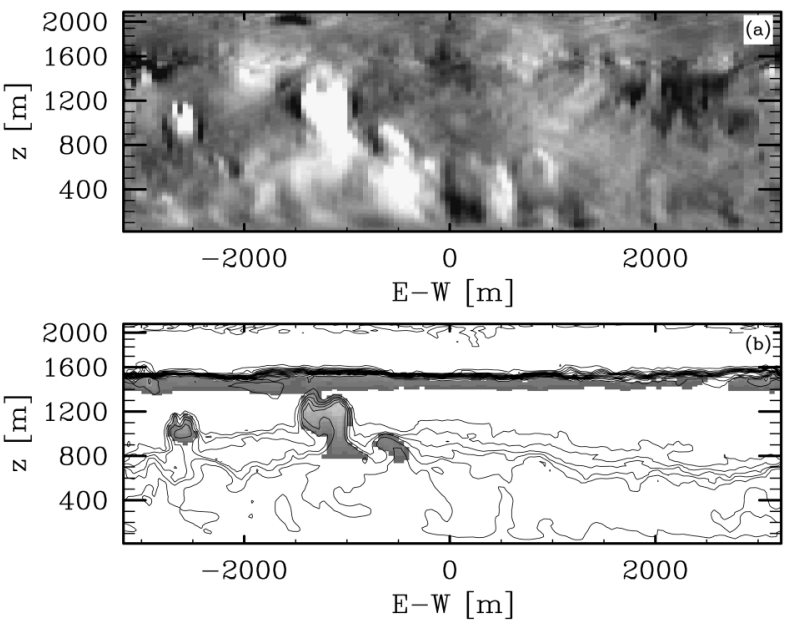

FIG. 6. The $x-z$ cross sections corresponding to Fig. 5 at $y=-750$ $\mathrm{m}$ : (a) raster-shaded (as in previous figure) vertical velocity, and (b) contours of $q_{t}$ evenly spaced every $0.5 \mathrm{~g} \mathrm{~kg}^{-1}$ and liquid water shaded.

cloud layer, although the tilting of the convective cores out of the plane makes this hard to see simply by looking at vertical correlations in the $w$ field in the plane. The connection between the cloud and subcloud layer is perhaps better illustrated in the cross-section plot (Fig. 6), although here too tilting out of the plane obscures the picture.

In addition to illustrating that the most convective elements are strongly rooted in the subcloud layer, Fig. 6 reinforces some previous findings. First, Fig. 6a further illustrates how the circulations in the cloud and subcloud layer differ. In the cloud layer intermittent structures dominate the transport. These events have large vertical velocities, are compensated by narrow regions of compensating flow in a sheath surrounding the clouds, and by gentle, rather larger-scale, subsidence throughout the surrounding domain-see, for instance, the region of dark shading (signifying downward motion) in the cloud layer at $x=2500$ in Fig. 6a. This is in contrast to the subcloud layer circulations, which are less intermittent, with updrafts and downdrafts being paired and commensurate in structure and intensity. Second, Fig. $6 \mathrm{~b}$ illustrates the instantaneous structure of the transition layer. Its pronounced structure is clearly evident in the contour of the $q_{t}$ field. A surprising and rather striking result is the extent of the vertical displacement of the transition layer; the upper contour of this layer varies over 400-m height (cf. the moisture contours at $x=0$, which are being lifted in association with convection just to the left vs at $x=2500 \mathrm{~m}$ where subsiding motion in the cloud layer compresses the transition layer).

\section{e. Cloud layer diagnostics}

As part of the standard diagnostics from the simulations, conditional averages in the cloud layer were performed. The averages were conditioned on either a grid point being cloudy, or on what we call cloud cores, that is the subset of cloudy points in positively buoyant updrafts. Formally, the core average of some variable $\psi(x, y, z, t)$ is defined as follows

$$
\Psi_{c l}(z) \equiv\langle\psi \mid I>0\rangle,
$$

where

$$
I(x, y, z, t)= \begin{cases}1 & q_{l}, w, \theta_{v}^{\prime}>0 \\ 0 & \text { otherwise }\end{cases}
$$

where in this one case angle brackets denote an average over horizontal surfaces and time, and $\langle\psi \mid I\rangle$ should be read as the average of $\psi$ conditioned on $I$. This method of sampling the flow is drawn from earlier work (e.g., Siebesma and Cuijpers 1995), and similarly we find that this latter form of sampling [represented by Eq. (9) above] is more effective than others sampling strategies we have tried. Figure 7 illustrates the results of this sampling for important thermodynamic fields.

The cloud-core points cover less than $4 \%$ of the domain at cloud base, with the area coverage decreasing through the lower half of the cloud layer. This view of the convective elements is consistent with our prior discussion of the skewed distribution of vertical velocities in the cloud layer. Overall the buoyant updrafts in the cloud make up between one-third and one-half of the cloudy points, with this fraction decreasing in the stratiform region.

Below $1100 \mathrm{~m}$ the conditionally averaged fields largely follow the pattern of previous analyses (e.g., Sie; Siebesma and Cuijpers 1995). That is thermodynamic fields tend to change linearly with height somewhat less steeply than the environmental values, vertical velocities increase with height, as does the buoyancy of parcels, while the mass flux follows the tendency of cloud coverage to decrease with height. Above $1100 \mathrm{~m}$ the effect of the stratiform layer becomes increasingly evident in the averages. As the sampling becomes increasingly affected by the stratiform layer an increasing fraction of the domain is sampled. That is, the relatively smallscale, radiatively driven circulations and their associated properties are increasingly projected onto conditionalaverage-based estimates of the cumulus properties. As a result the cloud averaged fields tend to be biased toward the environmental value above $1100 \mathrm{~m}$.

We can revise our sampling criteria to more successfully sample circulations thought to be associated with the cumulus layer. If we more restrictively define the cloud core as those points where the liquid water content is at least half as large as would be expected in a nonentraining adiabatic parcel ascending from cloud base to the given level, we find that the effect of the stratiform layer is strongly mitigated. This more restricted definition of the cloud core (not shown) gives a view of the cloud layer that does not differ substantially from previous analyses of shallow cumulus layers 

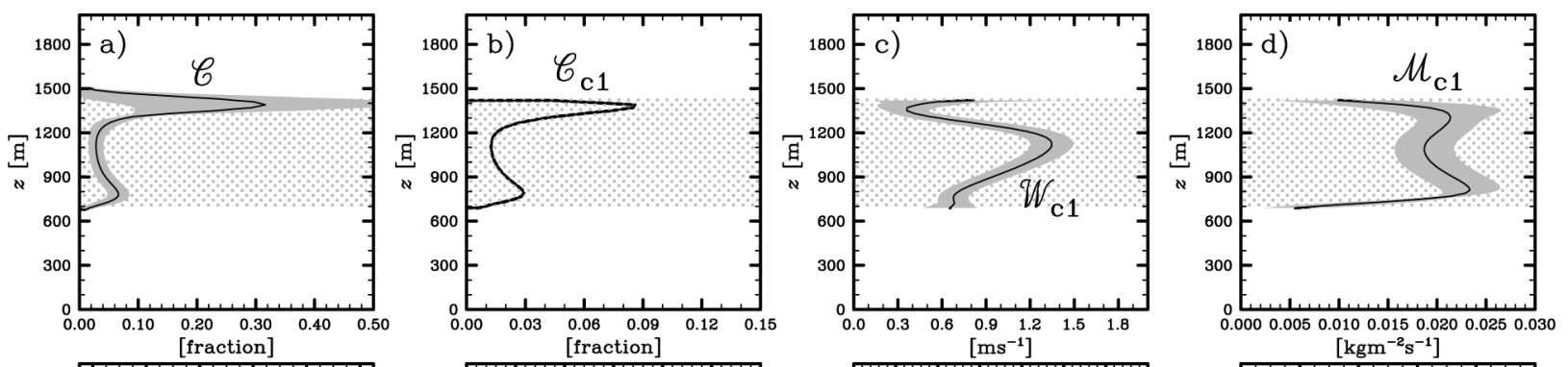

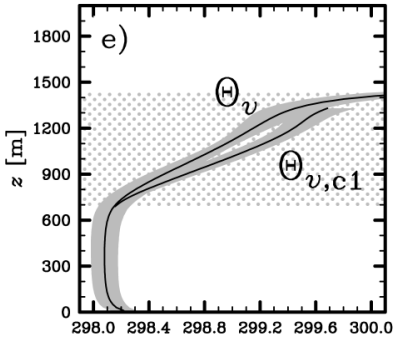

[K]

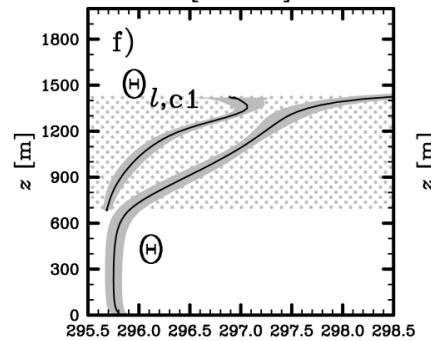

$[\mathrm{K}]$

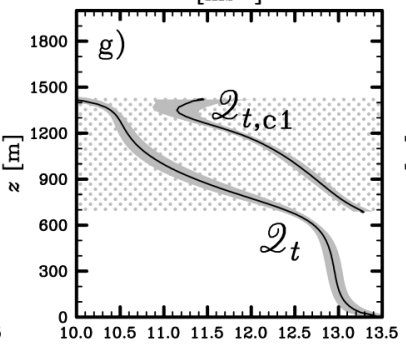

$\left[\mathrm{gkg}^{-1}\right]$

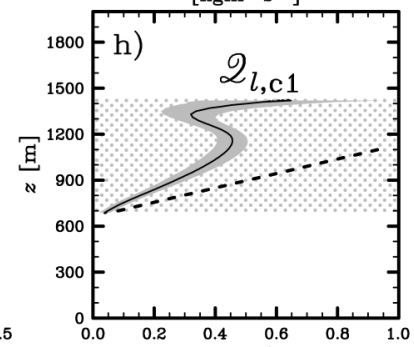

$\left[\mathrm{gkg}^{-1}\right]$

FIG. 7. Conditionally sampled cloud statistics: (a) cloud fraction; (b) cloud-core fraction; (c) cloud-core-averaged updraft velocity; (d) cloud-core mass flux; (e) cloud-core virtual potential temperature; (f) cloud-core $\theta_{i}$; (g) cloud-core $q_{t}$; (h) cloud-core $q_{l}$. For reference, mean profiles are also shown in (e)-(g), and in (h) the adiabatic liquid water content is also indicated by the dashed line. Results from the DHARMA calculation were not available for inclusion in (d)-(f).

without capping stratiform layers. This analysis suggests that the dynamical interaction between the stratiform layer and the cumulus layer may be secondary, and as a first approximation their respective dynamics can be considered to be independent of one another.

Last, we note the extent to which buoyant updrafts at cloud base reflect the surface properties (e.g., Figs. $7 \mathrm{e}-\mathrm{g})$. Figure $7 \mathrm{~g}$ in particular shows that total water mixing ratios at cloud base are consistent with the environmental values at $100 \mathrm{~m}$ or below. This view of the clouds being rooted in the surface layer is confirmed by the flow visualization (e.g., Fig. 6b).

\section{Discussion}

\section{a. Further sensitivities}

\section{1) Resolution, numerics, ANd SGS Models}

The UCLA and UKMO models were used to explore the sensitivity of the calculations to one's choice of numerical algorithms and SGS models as well as the size of the discretization mesh. In addition to the supplementary calculations listed in Table 2 a great variety of simulations (about 20) were performed using the UCLA model at standard resolution, but with minor changes to the SGS model or the numerics. By and large these calculations yielded few surprises. Thus, rather than attempting to systematically discuss them all we simply state our most important findings.

There is a clear tendency for cloud fraction $C$ to increase as the numerical mesh is refined. This is evident in Table 2 as well as from plots of liquid water path (e.g., $\mathcal{L}$ in Fig. 8 ), which is a better (i.e., unbounded) measure of stratiform cloud amount. Also evident in Fig. 8 is the tendency for cloud-base values of $C$ to be rather similar among the simulations, which reflects the general trend for the sensitivities to be most pronounced at the top of the cloud layer. Because the SGS formulation in the UKMO calculations sets the filter scale $\lambda$ proportional to the horizontal mesh size (i.e., $\lambda \propto \Delta x$ ), the factor of 5 refinement in the UKMO horizontal mesh corresponds to a factor of 5 refinement in $\lambda$. However, in the UCLA calculations $\lambda \propto(\Delta x \Delta y \Delta z)^{1 / 3}$, hence a factor of 2 refinement in the UCLA calculations only corresponds to a factor of 1.6 refinement in $\lambda$. For this reason we might expect the sensitivities in the UKMO calculations to be commensurately bigger; however, this only ends up being true for Fig. 8a.

Thus it is not surprising that (as shown in Fig. 8) the sensitivity of the calculations to the refinement depends in part on ones underlying numerical algorithms. The UCLA calculations with monotone scalar differencing are rather insensitive to the modifications in $\lambda$, indeed if one examined these results alone one might claim convergence. The results with centered differencing of scalars show a very sharp sensitivity (domain-averaged liquid water path is almost doubled in going from simulation B to D). Similarly, the UKMO simulations (which always maintain monotone scalar advection, but modify the momentum differencing) show that the calculations with forward-in-time (and upwinded) momentum are more sensitive (in this measure) to refinement. As we discuss later, part of the apparent sensitivity to different schemes is amplified by positive feedbacks associated with longwave radiative cooling at cloud top. Nonetheless, we show how, at least in this regime, ap- 

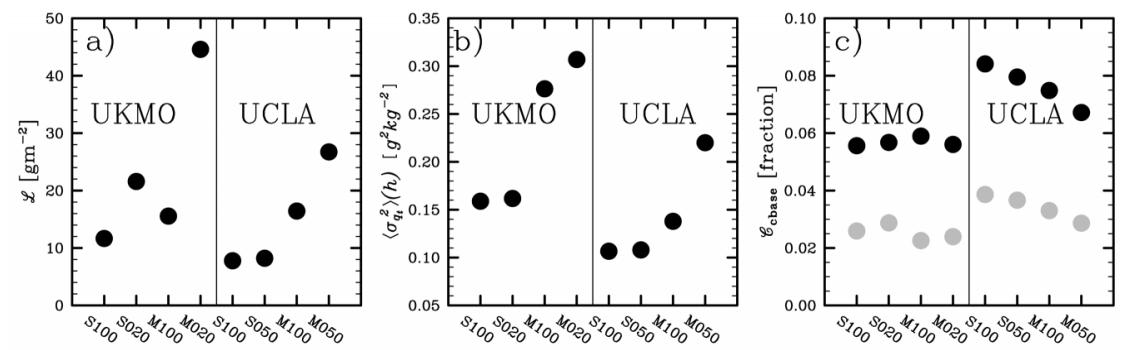

FIG. 8. Scalar measures from UKMO and UCLA simulations A-D. Note the ordering of the simulations: the last three numbers correspond to the horizontal grid spacing, the first letter denotes standard or modified (monotone) advection. Thus the simulations are ordered ACBD. (a) The liquid water path (cf. Fig. 2d) averaged over analysis period. (b) The local cloud-base maximum of $\sigma_{q}^{2}$. (c) The cloud-base maximum cloud (dark circles) and cloud-core amounts (light circles).

parent convergence in one calculation (cf. UCLA-A and UCLA-D) can be misleading, and how in some measures, simulations with different numerics appear to converge to different answers (cf. the UKMO simulations in Fig. 8b).

In the discussion of section $3 \mathrm{c}$ we noted that $\sigma_{q_{t}}$. was consistently larger for simulations with forward-in-time (and upwinded) momentum advection. Figure 8b shows a tendency for $\mathcal{L}$ and $\sigma_{q_{t}}(h)$ to be correlated, particularly in the UCLA calculations. This correlation is physically consistent with increased cloudiness corresponding to more turbulent mixing in the cloud layer, more entrainment drying, and a sharper differentiation between subcloud- and cloud-layer values of $q_{t}$. Nonetheless not all the differences can be attributed to physical processes: the simulations with forward-in-time (and upwinded) momentum advection consistently predict larger values of $\sigma_{q_{t}}(h)$, even when compared to the subset of simulations based on centered methods that predicted similar or greater cloud amounts (cf., DHARMA and UCLAD vs UKMO-B).

Despite some clear trends (e.g., there seems to be a significant negative correlation between $\mathcal{L}$ and $C$ at $z=$ $h$ ), statistics that reflect the energetics and the structure of the cumulus layer seem much less sensitive to the representation of small-scale features in the flow. In Fig. $8 \mathrm{c}$ the lightly shaded points illustrate how little cloud fraction at cloud base differs among the simulations. Repeating the analysis of Fig. 7 indicates that similarly small differences (especially below $1100 \mathrm{~m}$ where the influence of the stratiform cloud layer is reduced) are evident in other measures of the structure of the cumulus layer.

\section{2) Initial state}

Although a transition layer appears to be a ubiquitous feature of the trade cumulus regime, we know of no other simulations that explicitly incorporate its structure in the initial data. The question naturally arises as to the importance of such small-scale features. To explore this question we repeated the UCLA-A simulation, but eliminated the transition layer by removing the sounding point at $750-\mathrm{m}$ from the initial data in Table 1. Doing so results in a slightly more stable and moister cloud layer.

Broadly speaking such a modification to the initial data had a remarkably small impact on the overall simulation. The most notable impact of such a change was a slightly more rapid development of the cumulus layer during the spinup. However by the time of the analysis the simulation with the modified sounding was essentially indistinguishable from the five members of the UCLA-A ensemble. The remarkably small change underscores our general experience with LES, namely, that it is rather insensitive to rather small changes in the initial data. In some respects this is an expected and desirable feature that underscores the statistical nature of the tool. These results also emphasize that the transition layer is a signature of the flow itself, and it need (or should) not be specified in the initial data.

In addition to this test, one other was performed to evaluate the effect of the moisture forcing. Because of some confusion with the initial specifications, some groups did not include the height dependence in Eq. (5). Although this alone could not explain the variation in cloud amount among the simulations, we explored what effect this would have in a simulation with the UCLAA configuration. As might be expected the enhanced drying in the cloud layer in the sensitivity simulation leads to a reduction in cloud amount, that is, $C$ falls to 0.16 compared to the the ensemble estimate of $0.20 \pm$ 0.01 .

\section{3) Surface Layer}

As mentioned in section 2a the surface-flux prescription was awkward. To explore the impact of this aspect of the initial prescription we performed a simulation identical to UCLA-C, except using full surface-layer similarity theory, including a Charnock-like model of surface roughness, that is, $z_{0}=0.016\left(u_{*}^{2} / g\right)$. The principal impact of this modification was to reduce the value of $u_{*}$ (from the imposed value of 0.3 to a new value of 

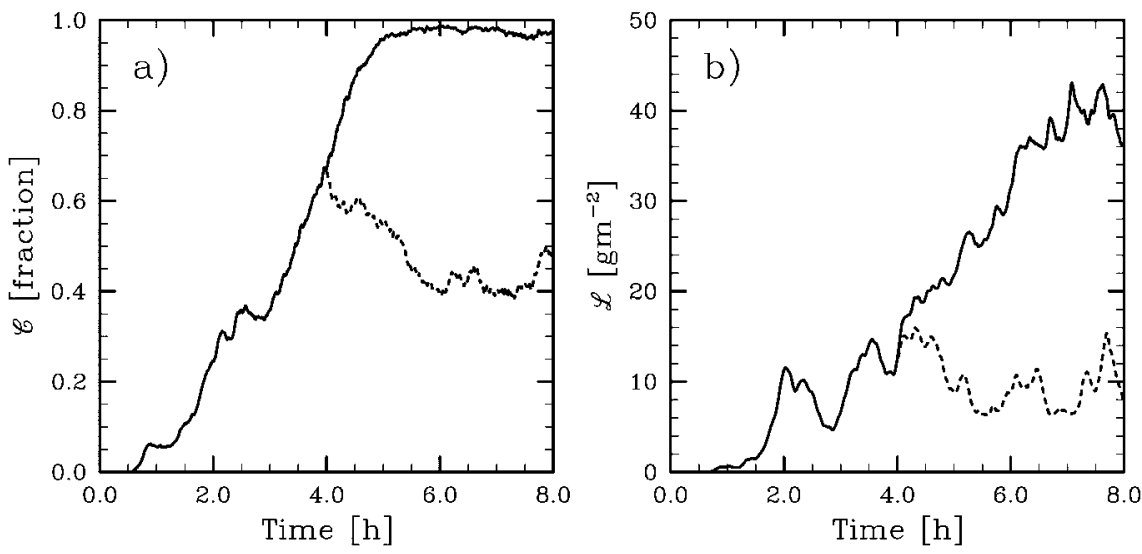

FIG. 9. (a) Time series of cloud fraction and (b) domain-averaged liquid water path for the UCLA-D (solid) calculation, and sensitivity run (short dashes) branched at $4 \mathrm{~h}$ with no radiative forcing.

0.21 ) by allowing it to respond to a reduction of the near-surface winds. This in turn led to less drag and smaller subcloud velocity deficits. As a result, near-surface winds and thermodynamic fluxes increased (e.g., surface buoyancy fluxes increased $36 \%$ ) relative to the standard simulations.

Reductions in the value of $u^{*}$ and increases in surface buoyancy fluxes (with little change in cloud-base height) lead to a more convectively driven subcloud layer. Over the last $3 \mathrm{~h}$ of the simulation, $-h / L$ (where $L$ is the Obukhov length) increased from 4 to 15 in response to the aforementioned changes. Flow analysis indicates that this change was sufficient to change the subcloudlayer turbulence structure from a streaklike to a more plumelike regime. A transition from boundary layer rolls to plumes is also observed in nature at similar values of $-h / L$ (LeMone 1980).

\section{4) RAdiAtive FEEDBACK}

How important is the radiative forcing in the development of the cloud layer? Figure 9 compares time series of cloud fraction and liquid water path from the UCLA-D calculation with those produced by a sensitivity calculation branched after $14400 \mathrm{~s}$. In the sensitivity calculation the interactive radiative forcing, as given by Eq. (6), was not applied. In response, $C$ immediately declined, and equilibrated at a considerably reduced level relative to the control, and $\mathcal{L}$ also declined significantly. This result indicates that the development of a nearly overcast stratiform layer in the standard UCLA-D calculation can partly be attributed to a positive feedback involving longwave radiative cooling from the top of an incipient stratiform cloud layer. The tendency for shortwave radiative effects to offset longwave radiative forcings suggests that the observed diurnal cycle in cloud fraction can be largely explained through radiative effects.

Figure 2 suggests that mean liquid water paths tended to range between 5 and $15 \mathrm{~g} \mathrm{~m}^{-2}$ among the calculations. Across this range of values the radiative cooling at cloud top would approximately double, from 36 to $64 \mathrm{~W} \mathrm{~m}^{-2}$. Thus if certain choices in numerical algorithms were more favorable for stratiform cloud development, such differences can be greatly amplified by radiative processes. In simulations of clouds observed during BOMEX (SIE), wherein this feedback is removed, simulations by different groups tend to be in better accord. From this, we conclude that the development of a stratiform cloud layer at cloud top is very sensitive to radiative processes, and that this sensitivity amplifies otherwise smaller differences among the simulations.

\section{b. Parameterization}

\section{1) TOP HAT MODELS}

One of the main motivations for this study was to explore whether parametric relationships, which were found to work well in other low cloud-fraction regimes, also described the behavior of cumulus clouds in this intermediate regime. In this regard there was a specific desire to test the generality of the findings by Siebesma and Cuijpers (1995). In that study they found that a mass-flux model well represents the simulated cloud layer, but that to do so commonly used parameters in the model had to be revised by an order of magnitude.

The mass-flux model they tested follows from the assumption that cloud-layer thermodynamics properties are distributed following a bi-delta distribution function. That is, they assume that the probability $p$ of measuring the value $\phi$ is

$$
p(\phi)=a \delta\left(\phi-\phi_{c}\right)+(1-a) \delta\left(\phi-\phi_{e}\right),
$$

where $\phi_{c}$ is the value of $\phi$ averaged over all the convective elements, $\phi_{e}$ is the mean value over the rest of the domain, and $a$ denotes the fractional coverage of convective elements. If $w$ is also described by such a 

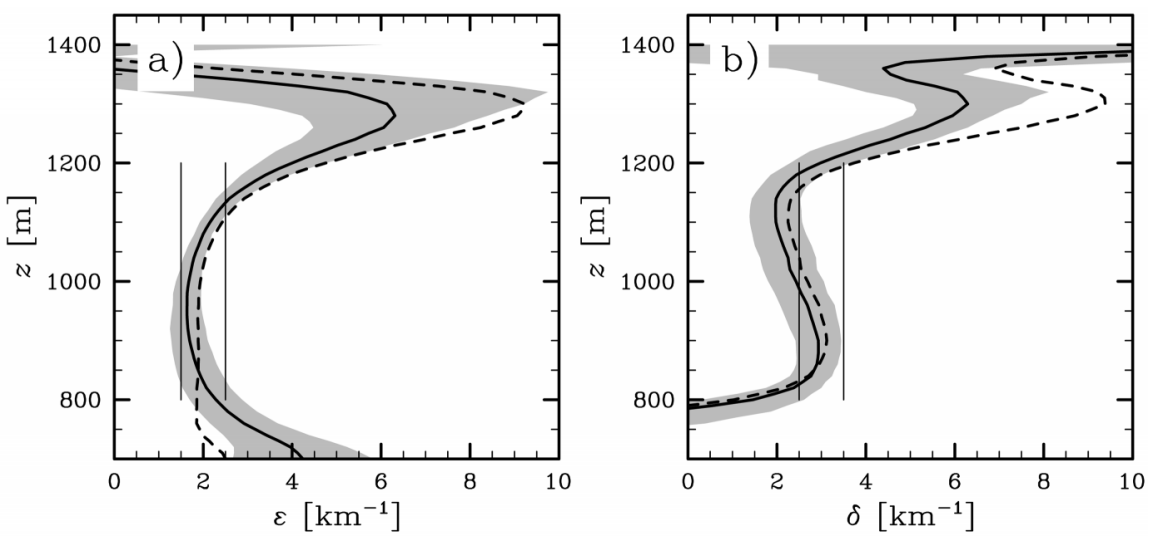

FIG. 10. Estimates of (a) fractional entrainment and (b) detrainment rates. Thick solid lines and shading are derived from $q_{t}$ budget. Dashed lines are from $\theta_{l}$ budget. Thin vertical lines indicate range of estimates from Siebesma (1998).

distribution, the flux for a resting mean state (i.e., $\bar{w}=$ 0 ) is automatically given as

$$
\overline{w^{\prime} \phi^{\prime}}=a w_{c}\left(\phi_{c}-\phi_{e}\right) \text {. }
$$

This is simply the product of the convective mass-flux $M$ and the difference between convective and environmental properties. ${ }^{3}$ Previous studies have found that such a model works well when convective elements are defined as those points that are both positively buoyant and cloudy, that is, what we call cloud-core points above.

Under the above assumptions, in the limit as $a \rightarrow 0$ but $M$ remains constant, we arrive at Tiedtke's (1989) mass-flux equations:

$$
\begin{aligned}
& \frac{\partial M}{\partial z}=E-D, \\
& \frac{\partial \phi_{c}}{\partial z}=\frac{E}{M}\left(\bar{\phi}-\phi_{c}\right), \\
& \frac{\partial \bar{\phi}}{\partial t}=-\frac{\partial}{\partial z}\left[M\left(\phi_{c}-\bar{\phi}\right)\right] .
\end{aligned}
$$

Here $E$ and $D$ stand for entrainment and detrainment, thereby representing the exchange of material properties across a contact surface separating the convective elements from the environment. Typically they are modeled as

$$
E=\epsilon M, \quad D=\delta M,
$$

where $\epsilon$ and $\delta$ are inverse length scales. These inverse length scales can be derived on the basis of auxiliary hypotheses (e.g., Siebesma 1998), although similarity arguments are often used to argue that they are universal

\footnotetext{
${ }^{3}$ Technically, what we call $M$ is the volume flux, while the mass flux is simply $\rho M$, but because we are considering effectively Boussinesq fluids the density does not enter into our derivations in any interesting way.
}

constants. Previous simulations suggest that $\delta$ is systematically larger than $\epsilon$ but that both are $O\left(1 / z_{i}\right)$.

We can test this model: first, by evaluating whether or not $\epsilon$ and $\delta$ are robustly predicted by different simulations and are consistent with previous estimates; and second, by exploring the ability of Eqs. (12)-(15) to represent the dynamics of the simulations given cloudbase estimates of both $M$ and $\phi_{c}$.

The results from the first test are shown in Fig. 10. Here $\epsilon$ and $\delta$ are diagnosed as

$$
\epsilon=\frac{1}{\bar{\phi}-\phi_{c}}\left(\frac{d \phi_{c}}{d z}\right), \quad \delta=\epsilon-\frac{1}{M} \frac{d M}{d z} .
$$

The above simple relations are consistent with Eqs. (12)-(13) and yield a rather accurate estimate of $\epsilon$ and $\delta$ [whose exact values can be calculated from rather more complicated relationships derived from cloud boundary budgets (e.g., Siebesma and Cuijpers 1995)]. For $\phi \in\left\{\theta_{l}, q_{t}\right\}$ we find that through the bulk of the cloud layer $\epsilon$ and $\delta$ are relatively insensitive to their basis of diagnosis (i.e., whether they were derived from the $\theta_{l}$ or $q_{t}$ fields) and are also reasonably consistent with previous estimates. Disagreement between previous estimates and diagnoses become increasingly evident toward cloud top, but this is thought to reflect the effect of the radiatively driven stratiform layer.

For the second test we evaluate Eqs. (12)-(14) given $\langle M\rangle$ and $\phi_{c}=\left\langle\left(q_{t}\right)_{c}\right\rangle$ at $800 \mathrm{~m}$ and $(\epsilon, \delta-1)=2 \mathrm{~km}^{-1}$ as estimated from Fig. 10. The simple model is able to capture much of the flux of the cloud layer (e.g., Fig. 11). Reducing $\epsilon$ by $25 \%$ but maintaining $\delta-\epsilon=1$ $\mathrm{km}^{-1}$ results in slightly improved agreement, particularly in the cloud profile in the lower part of the cloud layer. The disagreement between the mass flux and the cloud profiles in the upper part of the cloud layer predominantly reflects the failing of the cloud-core sampling. Refined sampling of the UCLA calculations (cf. section 3e) leads to profiles more like those predicted by the simple model. 

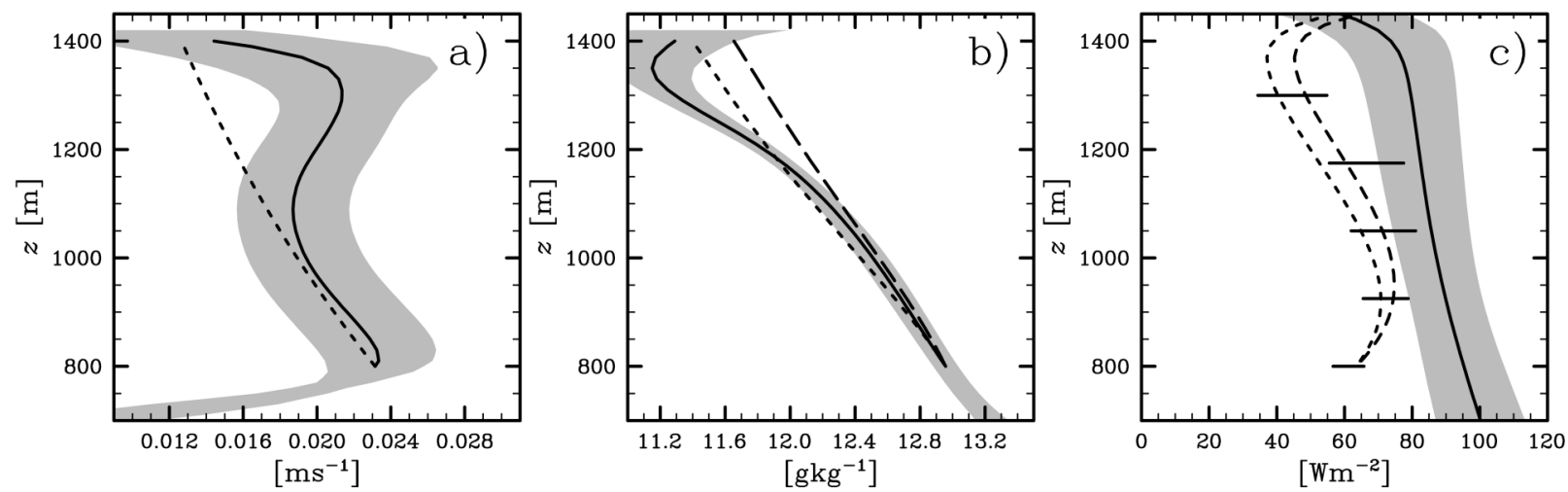

Fig. 11. (a) Mass flux, (b) $q_{t}$ in cloud, and (c) $\mathcal{F}_{L}$ from Eqs. (12)-(14). Solid banded lines show values derived from simulations. Estimates are for $\delta-\epsilon=1 \mathrm{~km}^{-1}$, with $\epsilon=2 \mathrm{~km}^{-1}$ (dotted) and $\epsilon=1.5 \mathrm{~km}^{-1}$ (dashed). Note that these yield identical predictions of $\mathcal{M}$ in (a). (c) The directly calculated mass-flux estimate of the flux, which we estimate from conditionally sampled fields from the different simulations, is also shown by the horizontal bars at five heights (cf. Fig. 3).

The model could be further adjusted to capture even more of the flux (i.e., by reducing $\delta$ relative to $\epsilon$ ) although it is unclear to what extent such fine-tuning is warranted. Irrespective of fine-tuning the simple massflux model tends to predict fluxes that fall off too sharply with height. This, however, might not be a failing of the model itself, as it may simply suggest that local circulations play a significant role in carrying the flux in the vicinity of the developing stratiform layer.

Although the model well represents the fluxes this is not (as pointed out by Wang and Stevens 2000) necessarily a critical test of the underlying probability density functions (PDFs). Deviations of the actual PDFs from bi-delta distributions can be large, but need not project onto the flux. In this case the assumption of bidelta PDFs can lead to large errors in estimates of the variance (where the Tiedtke limit predicts zero variance), but a rather good model of the flux.

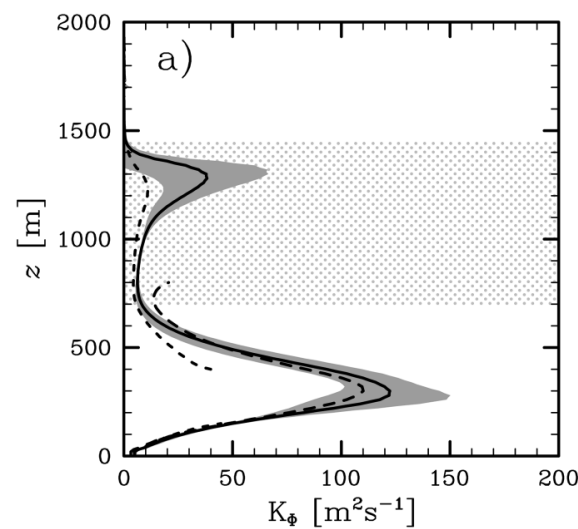

\section{2) EDDY-DIFFUSIVITY MODELS}

It is also instructive to interpret the simulations using a mixing length model of the fluxes, for example:

$$
K_{\Phi} \equiv-\overline{w^{\prime} \phi^{\prime}}\left(\frac{\partial \Phi}{\partial z}\right)^{-1}
$$

Here we diagnose the value of the exchange coefficient $\left(K_{\Phi}\right)$ necessary to relate the simulated fluxes of $\Phi$ for $\Phi \in\left(\Theta_{l}, Q_{t}, U, V\right)$. As is evident in Fig. 12 only $K_{Q_{t}}$ is well behaved throughout the layer.

Defining an exchange coefficient for $\Theta$ (Note that because the cloud fractions are so small the differences between $\theta_{l}$ and $\theta$ tend to be negligible.) is more problematic. In the subcloud layer $\partial \Theta / \partial z$ vanishes below the point where $\overline{\theta^{\prime} w^{\prime}}$ changes sign. This leads to a singularity in $K_{\Theta}$, similar to what is commonly found in cloud-free convective boundary layers (e.g., Ertel 1942;

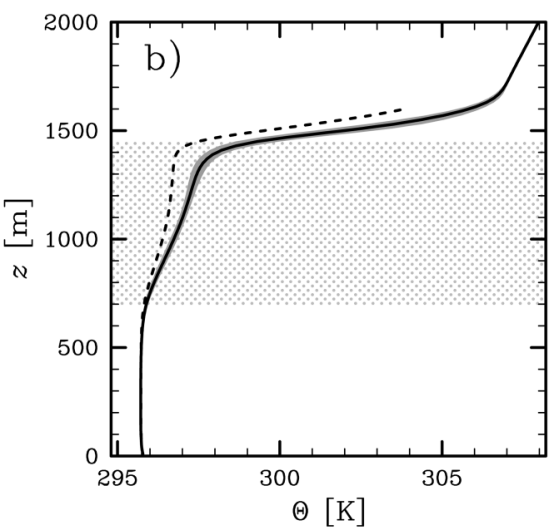

FIG. 12. (a) Implied exchange coefficients $\left\langle K_{\Phi}\right\rangle$, for $\Phi=Q_{t}$ (solid line and shading), $\Phi=U$ (dashed), and $\Phi=\Theta$ (short-dash). (b) $\Theta(z)$ (solid), $\Theta$ (short-dash) where $d \tilde{\Theta} / d z \equiv-\overline{w^{\prime} \theta^{\prime}} /$ $K_{O}$. Note that $K_{\Phi}$ is the average of $K_{\Phi} \mathrm{s}$ implied by each simulation (excepting DHARMA, INM, and KNMI). The KNMI and INM results were not included because (consistent with the $\overline{w^{\prime} w^{\prime}}$ biases by these models) their implied values of $K_{\Phi}$ were much larger in the subcloud layer (with $K_{\Theta}$ vanishing as low as $z=100 \mathrm{~m}$ ). The DHARMA result was excluded because it predicted values of $K_{\Phi}$ an order of magnitude larger at the top of the cloud layer. 
Holtslag and Moeng 1991; Wyngaard et al. 1991; and many others) over land. The exact point of the singularity varies among the simulations, so that $\left\langle K_{\Theta}\right\rangle$ is undefined through a significant region of the subcloud layer. Away from the singularity $K_{\Theta}$ behaves similarly to $K_{Q_{t}}$ although it is substantially smaller in the upper part of the cloud layer. That $K_{\Theta}$ and $K_{Q_{t}}$ are not identical in regions where $K_{\Theta}$ is well defined is perhaps to be expected given the different flux geometries (i.e., the ratio of their bottom-up to top-down components) of the two scalars, the skewness of the flow, and previous results that suggest that in asymmetrically forced flows the mixing of scalars originating from either boundary is also asymmetric (Wyngaard and Brost 1984).

The singularity in $K_{\Theta}$ is a well known and dramatic failing of the local model of the fluxes implied by Eq. (17). But is it really that significant? To put things in a different light we ask, given specified boundary fluxes, and using the $K_{Q_{t}}$ profile to describe the mixing of all scalars, how much in error will the quasi-steady profile of $\Theta$ be? The answer is given in Fig. 12b, where we plot the implied quasi-steady profile of $\Theta$ alongside the consensus profile. Despite rather large differences between $K_{Q_{t}}$ and $K_{\Theta}$ in the subcloud layer, these differences have rather insignificant effects on the resultant profiles. The much more substantial error comes from attempting to carry the model through the cloud layer and into the trade inversion. Here not accounting for differences between $K_{\Theta}$ and $K_{Q_{t}}$ leads to a cloud layer that is too well mixed in $\Theta$.

We have also looked at the exchange coefficients for momentum. An exchange coefficient for the dominant component of the wind $K_{U}$ is well behaved in the subcloud layer (dashed line in Fig. 12a) and commensurate with $K_{Q_{t}}$. But because the simulated profile of $U$ has an extremum near $900 \mathrm{~m}$ while $\overline{u^{\prime} w^{\prime}}$ is positive definite, $K_{U}$ behaves poorly in the cloud layer. The shape of the wind profiles, and in particular the extremum in $U$ [which is also evident in observed wind profiles (Brümmer et al. 1974)], is a consequence of the rather inefficient mixing of momentum in the cloud layer, and the baroclinicity implied by the height variation of the geostrophic wind. Although the magnitude of this extremum is to some extent an artifact of our surface forcing, the previously discussed simulations (wherein $u_{*}$ was allowed to vary freely) still retain this feature. Simulations of the dry-convective boundary layer efficiently mix momentum through the entire boundary layer, so if a local extremum develops, it does so at the top of the PBL where the momentum flux vanishes. We speculate that this difference accounts for the failure of the eddy-exchange model in our case, despite being able to represent the mixing in the baroclinic dry convective boundary layer (Brown 1996).

In summary our analysis suggests that a local model of the fluxes (even in the absence of nonlocal corrections) can be a reasonable approximation to the simulated subcloud layer, but that the failings of the model become less tolerable in the cloud layer. Because the convective dynamics tend to relax the cloud layer to a state that is not effectively well mixed, our results suggest that using an exchange coefficient model of the fluxes through the entire boundary layer leads to significant imbalances in the heat and moisture profiles, and misrepresents the mixing of momentum above the subcloud layer. While some of the failings of the exchange coefficient model might be mitigated by accounting for the flux geometry and thereby constructing different profiles of $K_{\Phi}$ for each $\Phi$, such a model will still not properly represent the mixing of momentum.

\section{3) Closure assumptions}

Our previous analysis indicates that however one models the interior of convective layers, the boundaries of these layers are poorly represented. This has motivated recent attempts to couple (or match) boundary layer models to explicit and distinct parameterizations both at the surface, and in the entrainment layer (Beljaars and Viterbo 1998). In the intermediate trade wind boundary layer a matching or consistency condition is also demanded at cloud base. Conditions for both regions are discussed below.

\section{(i) Cloud base: $z=h$}

We find that $h$ is remarkably stationary, irrespective of how it is defined. Indeed $d h / d t \approx 2 \mathrm{~mm} \mathrm{~s}^{-1}$, which is on the order of the large-scale subsidence velocity and an order of magnitude less than the cloud-core volume flux (cf. $\mathcal{M}$ in Fig. $7 \mathrm{~d}$ ). This difference suggests that to a good degree of approximation, the entrainment deepening of the subcloud layer is largely balanced by the evacuation of subcloud-layer mass by cumulus convection. Thus the assumption that $\mathcal{M}$ at cloud base is simply that necessary to keep the subcloud-layer lifting condensation level $z_{\mathrm{lcl}}$ at a fixed height (e.g., Albrecht et al. 1979) appears to be well supported by the simulations; although this might be an artifact of the rather constant ratio of surface heat to moisture fluxes, and the steadiness of the resultant circulations. During the daytime over land there are clear and pronounced diurnal variations in cloud-base height, and similar variations may occur over the ocean in rapidly evolving flows such as cold-air outbreaks.

\section{(ii) Cloud top: $z=z_{i}$}

In contrast to BOMEX, and other simulations of trade cumulus (e.g., Sommeria 1976, 1978; SIE), in the ATEX boundary layer most of the convergence in the moisture flux is confined to a very thin layer at the base of the trade inversion. This feature is evident in all the simulations, irrespective of stratiform cloud amount. It 

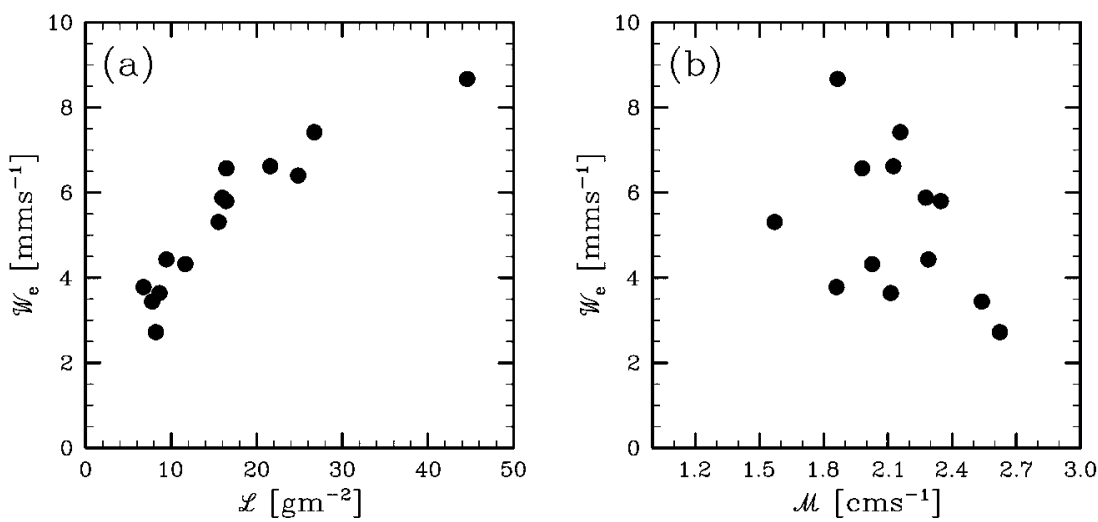

FIG. 13. Analyzed entrainment rates over the last $5 \mathrm{~h}$ of simulation vs (a) domain-averaged liquid water path and (b) maximum cloud-core volume flux below $1200 \mathrm{~m}$. All simulations excepting UCLA-E from Table 2 are plotted.

probably results from the very strong capping inversion that confines the detrainment of all clouds to an effectively thin layer, but may also result from a slightly more conditionally unstable cloud layer. In any case the presence of a thin layer near cloud top, where the fluxes rapidly go to zero, motivates an interfacial analysis similar to that done for stratocumulus or cloud-free convective boundary layers. In this approach, the inversion is idealized as a jump so that fluxes across the inversion can be simply represented as the product of an entrainment velocity $W_{e}$ and a jump $\Delta \Phi$ denoting the change in some variable $\Phi$ across the inversion.

Attempts to evaluate the behavior of $W_{e}$ in the simulations are clearly hampered by the varying degree of cloudiness among the simulations. For instance, Fig. 13a suggests that the entrainment rates at cloud top and the stratiform cloud amount are tightly coupled. As $\mathcal{L}$ increases by a factor of $6, W_{e}$ increases by a factor of 4 . In one sense this is counterintuitive. Should not enhanced entrainment of warm and dry air lead to a thinning of the cloud? Not necessarily. Because the increased entrainment is a result of enhanced radiative cooling, changes in the saturation deficit $\left(q_{t}-q_{s}\right)$ associated with entrainment warming and drying are offset by radiative cooling.

It is of interest (e.g., Wyant et al. 1997) to estimate how much, if at all, the cumulus clouds directly contribute to entrainment. The only way we can address this issue here is to attempt to try and subtract the stratiform effect out. If we assume that the influence of the stratiform layer is rather small for simulations where $\mathcal{L}$ $<10 \mathrm{~g} \mathrm{~m}^{-2}$, one can roughly attribute entrainment rates of $2-3 \mathrm{~mm} \mathrm{~s}^{-1}$ to the direct action of the cumulus clouds. ${ }^{4}$ These entrainment rates are on the order of what one would expect from a similarly forced dry convective boundary layer extending to $z_{i}$. A simple parameterization of cumulus-induced entrainment is

\footnotetext{
${ }^{4}$ Averaging only over the liquid water profile associated with the cumulus turrets (e.g., Fig. 3a) yields $\mathcal{L} \approx 6 \mathrm{~g} \mathrm{~m}^{-2}$.
}

$$
\frac{W_{e}}{\mathcal{M}}=A\left(\frac{b_{s}}{\Delta B}\right),
$$

where $\Delta B=(g / \Theta)\left[\Theta_{v}\left(z_{i}+\delta z\right)-\Theta_{v}\left(z_{i}-\delta z\right)\right]$ is the buoyancy jump across $z_{i}$ and $b_{s}$ is a measure of the cloud buoyancy. If we instead identify $b_{s}$ with the convective available potential energy divided by the cloud depth, this parameterization is identical to that proposed by Wyant et al. (1997). Our analysis suggests that if Eq. (18) is indeed valid, and if $b_{s}$ is taken as the buoyancy excess evident in Fig. 7e, then $A$ is between 2 and 8 .

Observations during ATEX indicate that $z_{i}$ varied by approximately $400 \mathrm{~m}$ over the course of the diurnal cycle (Augstein et al. 1974). What caused this variation? The two most obvious possibilities include a diurnal variation in the entrainment rate (driven by the cycle of solar heating) or a diurnal variation in the large-scale vertical velocity field. In our simulations the development of a stratiform layer increases the entrainment rates by up to $5-6 \mathrm{~mm} \mathrm{~s}^{-1}$. Thus during the night the enhanced entrainment associated with a more pronounced stratiform cloud layer might be able to explain a diurnal cycle in $z_{i}$ of up to $200 \mathrm{~m}$. Consequently, it appears that if the LES-derived entrainment rates are realistic, other processes must be invoked to explain the observed diurnal variation in $z_{i}$.

\section{Concluding remarks}

Our main points, and some that follow from them, are organized thematically below and discussed when possible in the context of the conceptual cartoon of Fig. 14.

\section{a. Basic structure of the intermediate regime}

The simulations provide a basis for synthesizing the turbulent structure with the observed mean vertical structure and large-scale forcings in an intermediate trade wind boundary layer regime. They show that given 


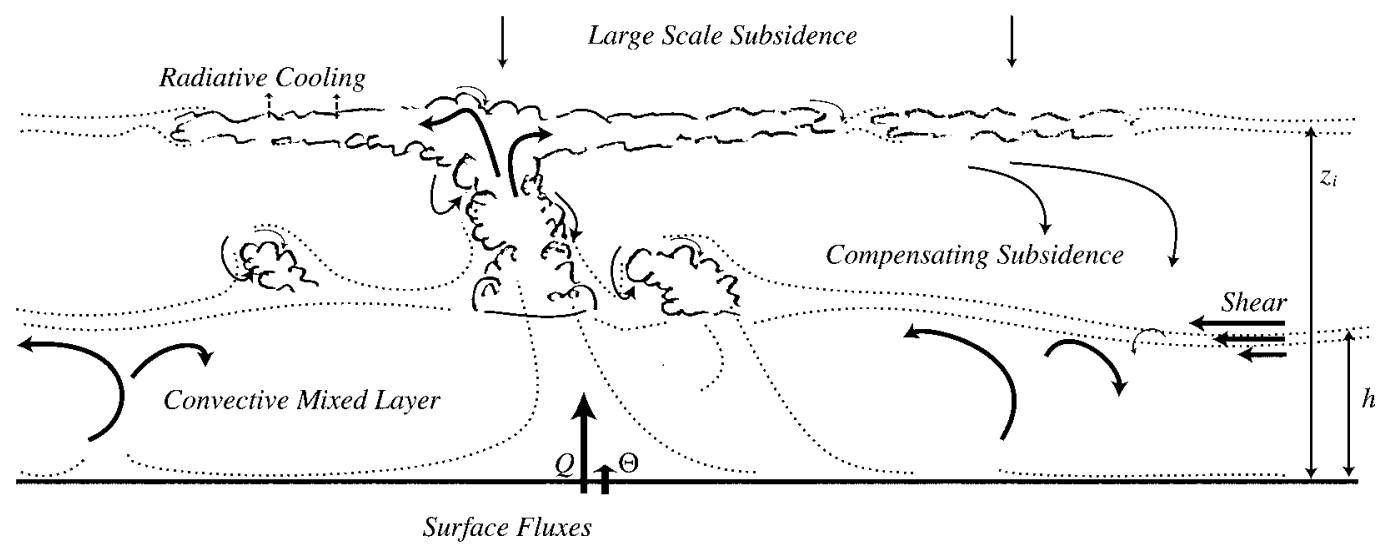

FIG. 14. Conceptual diagram of intermediate trade cumulus regime.

the observed forcings the observed thermodynamic state is compatible (or in balance) with the turbulent circulations that ensue. That is, the convective circulations that develop do not appreciably alter the mean vertical structure that was observed.

The convective circulation is distinctly organized in two main layers, an approximately 700-m-deep cumulus layer, rooted in a dry subcloud layer of approximately the same depth. Several sublayers are also identifiable: there is a detrainment layer around $1400 \mathrm{~m}$ in which stratiform clouds develop, a highly variable transition layer near cloud base, and a surface layer. The thermodynamic state in the PBL, extending from the surface and up to the thin stratiform layer, is effectively quasisteady. By this we mean that the thermodynamic fluxes are approximately linear with height, and thus the shape of the mean profiles are stationary. Although the thermodynamic structure of the cloud and subcloud layers is tightly coupled, there are sharp differences in the accompanying turbulent circulations. As has been noted many times previously, mixed-layer scaling provides a good description of the subcloud-layer turbulence. The simulations even show some organization in the pattern of subcloud circulations (i.e., streaks vs plumes) according to empirically determined rules. The cloud-layer turbulent structure, on the other hand, is characterized by more skewed circulations. Almost all of the transport is carried out by buoyant elements within the cloud and which occupy $<5 \%$ of the domain. The clouds themselves are deeply rooted not just in the subcloud layer, but in the surface layer. For instance, conditionally sampled cloud-base properties have specific humidities commensurate with those found in the surface layer.

Statistically, the cumulus clouds are remarkably similar to those observed in other regimes-particularly in the lower part of the cloud layer. For the most part their mass flux decreases with height. Buoyancy excesses in the cloud are relatively small $(\approx 0.2 \mathrm{~K})$ but increase with height. The specific humidity within the cloud is characteristic of some of the moistest air in the subcloud layer, while $\theta_{l}$ is characteristic of some of the coolest.
The average value of $q_{l}$ within convective cloudy elements tends to increase at about half its adiabatic value. In accordance with previous studies we note that the tendency of the mass flux is primarily a feature of an ensemble of clouds, and should not be interpreted as the tendency of any one cloud-whose fractional area might be more constant through its depth.

The principal differences between these simulations and previous ones are 1) the extent to which the cloud layer is in a quasi-steady state, and 2) the presence of a stratiform layer at the top of the cloud layer. Both differences seem to be the result of the relative strength of the trade inversion in the intermediate regime, which effectively caps all convective elements and inhibits entrainment drying. An alternative explanation that we have not explored is that the differences may be due also to the more conditionally unstable cloud layer in the current simulations (relative to their BOMEX counterparts). Regardless of the reason, efficient transport of moisture to the top of the cloud layer and the development of a stratiform cloud layer there promote local, radiatively driven, circulations, which in turn enhance mixing across the trade inversion and generate a better mixed upper cloud layer. These circulations are also less skewed and tend to be more reminiscent of stratocumulus.

\section{b. Sensitivities and convergence}

The simulations are ambiguous on the issue of sensitivities. Some aspects of the flow are remarkably robust, both across different simulations, and within a sequence of increasingly refined simulations based on a single algorithmic framework. The most apparent sensitivity of the simulations is their inability to give consistent predictions of stratiform cloud fractions. But there are others. For instance the sensitivity of $\sigma_{q_{t}}(h)$ is vexing, and the sensitivity of the surface profiles suggests that surface energy budgets from different models may differ appreciably. Some of the more robust aspects of the simulations include their representation of the 
lower cloud-layer dynamics, their prediction of convective scaling for the subcloud layer, and their representation of the differential mixing of the momentum in the cloud layer versus subcloud layer, respectively.

Different simulations disagree sharply on their prediction of stratiform cloud amount at the top of the cloud layer. This disagreement is real: ensembles of simulations produced by a single model predicted an order of magnitude less variability in this field than did an ensemble of different models. The inability of the models to duplicate estimates of cloud fraction is amplified by a positive feedback associated with the radiative forcing. The results clearly indicate that the development or breakup of stratiform cloud layers is the product of an intricate balance among large-scale forcings, turbulent dynamics, and the radiative forcings. Given both specified forcings and radiative algorithms, the deciding factor seems to be the numerical representation of the turbulent circulations. The effect of algorithmic differences (truncation errors if you will) persist even at relatively fine resolution. Moreover, apparent convergence in one set of calculations does not imply that another set of calculations, based on a different numerical framework, will also converge. Our results thus suggest that in this regime, and perhaps in any transitional regime, LES is at best a heuristic tool for evaluating the processes that govern the lifetime of stratiform cloud ensembles.

Another area of disagreement among the models is at the surface. Disagreements among simulations in this region of the flow is partly hidden because of the thinness of the surface layer, and because surface fluxes are often prescribed. However, most of the difference in surface fluxes among the models reflects differing predictions of the low-level wind structure. Thus the wellknown difficulty in matching LES to the surface is evident in these simulations, and suggests that attempting to use LES to derive things like geostrophic drag coefficients might be problematic.

Despite the rather large variability in the statistics at the top of the cloud layer the simulations provide a rather consistent picture of the cumulus cloud layer itself. For instance, cloud-base mass fluxes, thermodynamic properties, and area fraction are all remarkably consistent. Among these quantities the greatest differences among the models is in terms of their predictions of mass fluxes; however, such differences appear small relative to our level of ignorance regarding how best to improve low-dimensional representations of processes such as those we are simulating here.

\section{c. Simple models}

We have also attempted to use the information from the simulations to identify basic issues and test simple ideas associated with low-dimensional representations of the dynamics of the PBL. In general we find that mixing length models perform adequately in the subcloud layer. While their performance can be improved by including nonlocal terms, this seems to be a secondary effect.

In the middle and lower portions of the cloud layer, mass-flux models are also found to adequately describe the transport of heat and moisture, although we have not tested mass-flux representations of momentum transport. The mass-flux approach fails to represent the dynamics at the top of the cloud layer in at least two respects. Neither the enhancement of the local circulation in response to developing stratiform layers nor the interaction of the convective elements with the trade inversion is properly captured by the simple model we tested.

Because the thermodynamics of the cloud layer and subcloud layer are so tightly coupled, either the massflux approach has to be generalized to allow its extension into the subcloud layer or matching rules must be formulated to link the two different approaches at cloud base. In any case, our work suggests that the most important question in ongoing attempts to model layers such as those described herein amount to what one might call interface rules. By interface rules we mean a set of rules (based on the bulk properties of the flow) that describe how to consistently match both the top of the cloud layer to the free flow, and the subcloud layer to the cloud layer. The simulations provide some hints as to how to proceed. They indicate that cumulus convection on its own entrains air across a sharp interface relatively inefficiently, and they also reaffirm the fact that the cumulus layer must be coupled to a convectively scaled subcloud layer.

The simulations also provide insight into the transition layer. For the flux geometries studied here moisture was transported more efficiently than heat in the cloud layer, and as a result the transition layer was more evident in $q_{t}$ than in $\theta$. This transition layer was also highly variable, both in thickness and in height-tending to be lower and thinner away from the convecting regions. Thus the results support the idea that moist convection limits itself in part through the modulation of the height and thickness of the transition layer. Increased convective fluxes demand more compensating downward motion in the cloud layer, which in turn inhibits the development of convection elsewhere. This negative feedback may also be part of the reason why some cloudlayer statistics [such as $C(h), \mathcal{M}(h)$, etc.] are relatively robust across simulations. Last, the transition layer should be seen as a consequence of internal turbulent dynamics, and thus to impose it as an initial condition is neither necessary nor (in retrospect) warranted. The same might be said of the trade inversion, but the timescales of its development are sufficiently long so as to leave us with little choice.

\section{d. Further research}

One goal of this intercomparison is that by documenting the basic structure and sensitivities in the sim- 
ulated regime we can help guide future research, including field studies. One of the key scientific questions we had hoped to address in this study remains outstanding, namely, what relationship exists between cloud fraction and the mean state?

Because of substantial doubt regarding the fidelity of LES on this question, it is hoped that progress can be made on other fronts. In particular we envision that long-term field studies will incorporate remotely sensed cloud fields, in situ measurements of atmospheric vertical structure, and measurements of surface fluxes. Because of the subtlety involved, measurements from a site that do not appreciably disturb the flow are of paramount importance. As discussed by Stevens and Lenschow (2001) this strategy is based on the belief that statistical relationships based on carefully chosen and measured data derived from long-term sampling (perhaps from an inactive oil platform or a large moored buoy?) will be most useful in constraining the simulations. Such a measurement strategy is also well suited to further questions raised by the simulations. For instance,

- Can the simulations predict observed transitions from streaks to plumes with any degree of precision?

- To what extent can the simulations predict the cloud population statistics in the cloud layer?

- Are the statistics of the simulated transition layer (and the joint surface and transition layer statistics) in accord with those observed?

Yet a further advantage of such an approach is that it could provide a valuable dataset for the calibration of a new generation of space-borne cloud sensors such as CloudSat and PICASSO-CENA.

Acknowledgments. This work was supported in part by NSF Grant ATM-9985413. Phases of this project also benefited from support by the Alexander von Humboldt Foundation, the Advanced Study Program of NCAR, the World Climate Research Program, and European Union Project ENV4-CT95-0107. The comments of C. S. Bretherton, S. A. Klein, and three anonymous reviewers substantially improved the manuscript. The authors are especially grateful to the Instituto Nacional Meteorologia for graciously hosting the workshop. Last, we would like to emphasize that the results presented herein, as well as results from individual simulations, are available in electronic form upon request.

\section{REFERENCES}

Ackerman, A. S., O. B. Toon, and P. V. Hobbs, 1993: Dissipation of marine stratiform clouds and collapse of the marine boundary layer due to the depletion of cloud condensation nuclei by clouds. Science, 262, 226-229.

- - - D. E. Stevens, A. J. Heymsfeld, V. Ramanathan, and E. J. Welton, 2000: Reduction of tropical cloudiness by soot. Science, 288, 1042-1047.
Albrecht, B. A., 1989: Aerosols, cloud microphysics and fractional cloudiness. Science, 245, 1227-1230.

— 1991: Fractional cloudiness and cloud-top entrainment instability. J. Atmos. Sci., 48, 1519-1525.

, A. K. Betts, W. H. Schubert, and S. K. Cox, 1979: A model of the thermodynamic structure of the trade-wind boundary layer. Part I: Theoretical formulation and sensitivity tests. J. Atmos. Sci., 36, 90-98.

Augstein, E., H. Riehl, F. Ostapoff, and V. Wagner, 1973: Mass and energy transports in an undisturbed Atlantic trade-wind flow. Mon. Wea. Rev., 101, 101-111.

- , H. Schmidt, and F. Ostapoff, 1974: The vertical structure of the atmospheric planetary boundary layer in undisturbed trade winds over the Atlantic Ocean. Bound.-Layer Meteor., 6, 129150 .

Beljaars, A., and P. Viterbo, 1998: Role of the boundary layer in a numerical weather prediction model. Clear and Cloudy Boundary Layers, A. A. M. Holtslag and P. G. Duynkerke, Eds., Elsevier, 287-304.

Brown, A. R., 1996: Large-eddy simulation and parameterization of the baroclinic boundary layer. Quart. J. Roy. Meteor. Soc., 122, $1779-1798$

Brümmer, B., E. Augstein, and H. Riehl, 1974: On the low-level wind structure in the Atlantic trade. Quart. J. Roy. Meteor. Soc., 100, 109-121.

Duynkerke, P. G., and Coauthors, 2000: Intercomparison of threeand one-dimensional model simulations and aircraft observations of stratocumulus. Bound.-Layer Meteor., 92, 453-487.

Ertel, H., 1942: Der vertikale Turbulenz-Wärmestrom in der Atmosphäre. Meteor. Z., 59, 250-253.

Garstang, M., and A. K. Betts, 1974: A review of the tropical boundary layer and cumulus convection: Structure, parameterization, and modeling. Bull. Amer. Meteor. Soc., 55, 1195-1205.

Holtslag, A. A. M., and C.-H. Moeng, 1991: Eddy diffusivity and countergradient transport in the convective atmospheric boundary layer. J. Atmos. Sci., 48, 1690-1698.

LeMone, M., 1980: The marine boundary layer. Proc. Workshop on the Planetary Boundary Layer, Boulder, CO, Amer. Meteor. Soc., 182-231.

Riehl, H., C. Yeh, J. S. Malkus, and N. E. LaSeur, 1951: The northeast trade of the Pacific Ocean. Quart. J. Roy. Meteor. Soc., 77, 598-626.

Schmidt, H., and U. Schumann, 1989: Coherent structure of the convective boundary layer derived from large-eddy simulations. $J$. Fluid Mech., 200, 511-562.

Siebesma, A. P., 1998: Shallow Convection. Vol. 513, Buoyant Convection in Geophysical Flows, Kluwer Academic, 441-486.

- and J. W. M. Cuijpers, 1995: Evaluation of parametric assumptions for shallow cumulus convection. J. Atmos. Sci., 52, 650-666.

Sommeria, G., 1976: Three-dimensional simulation of turbulent processes in an undisturbed trade wind boundary layer. J. Atmos. Sci., 33, 216-241.

_ 1978: Direct testing of a three-dimensional model of the planetary boundary layer against experimental data. J. Atmos. Sci., 35, 25-39.

Stevens, B., and D. H. Lenschow, 2001: Observations, experiments, and large eddy simulation. Bull. Amer. Meteor. Soc., 82, 283294.

Stevens, D., and C. S. Bretherton, 1997: A new forward-in-time advection scheme and adaptive multilevel flow solver for nearly incompressible atmospheric flow. J. Comput. Phys., 129, 284295.

Tiedtke, M., 1989: A comprehensive mass flux scheme for cumulus parameterization in large-scale models. Mon. Wea. Rev., 117, $1779-1800$.

, W. A. Heckley, and J. Slingo, 1988: Tropical forecasting at ECMWF: The influence of physical parmetrization on the mean 
structure of forecasts and analyses. Quart. J. Roy. Meteor. Soc., 114, 639-964.

von Ficker, H., 1936: Die Passatinversion. Veröffentlichungen des Meteorologischen Institutes der Universität Berlin, Vol. 1, No. 4, $33 \mathrm{pp}$.

Wang, S., and B. Stevens, 2000: Top-hat representation of turbulence statistics in cloud-topped boundary layers: A large-eddy simulation study. J. Atmos. Sci., 57, 423-441.

Wyant, M. C., C. S. Bretherton, H. A. Rand, and D. E. Stevens, 1997: Numerical simulations and a conceptual model of the stratocumulus to trade cumulus transition. J. Atmos. Sci., 54, 168-192.
Wyngaard, J. C., and R. A. Brost, 1984: Top-down and bottom-up diffusion of a scalar in the convective boundary layer. J. Atmos. Sci., 41, 102-112.

_ C.-H. Moeng, and J. Weil, 1991: Parameterizing turbulent diffusion in the atmospheric boundary layer. Studies in Turbulence: In Recognition of Contribution by John Lumley, T. Gatski, C. Speziale, and S. Sarkar, Eds., Springer-Verlag, 3-16.

Yin, B., and B. Albrecht, 2000: Spatial variability of atmospheric boundary layer structure over the eastern equatorial Pacific. $J$. Climate, 13, 1574-1592. 